\title{
Search for Physics Beyond the Standard Model in Events with High-Momentum Higgs Bosons and Missing Transverse Momentum in Proton-Proton Collisions at $13 \mathrm{TeV}$
}

\author{
A. M. Sirunyan et al. \\ (CMS Collaboration)
}

(Received 22 December 2017; published 11 June 2018)

\begin{abstract}
A search for physics beyond the standard model in events with one or more high-momentum Higgs bosons, $H$, decaying to pairs of $b$ quarks in association with missing transverse momentum is presented. The data, corresponding to an integrated luminosity of $35.9 \mathrm{fb}^{-1}$, were collected with the CMS detector at the LHC in proton-proton collisions at the center-of-mass energy $\sqrt{s}=13 \mathrm{TeV}$. The analysis utilizes a new $b$ quark tagging technique based on jet substructure to identify jets from $H \rightarrow b \bar{b}$. Events are categorized by the multiplicity of $\mathrm{H}$-tagged jets, jet mass, and the missing transverse momentum. No significant deviation from standard model expectations is observed. In the context of supersymmetry (SUSY), limits on the cross sections of pair-produced gluinos are set, assuming that gluinos decay to quark pairs, $H$ (or $Z$ ), and the lightest SUSY particle, LSP, through an intermediate next-to-lightest SUSY particle, NLSP. With large mass splitting between the NLSP and LSP, and 100\% NLSP branching fraction to $H$, the lower limit on the gluino mass is found to be $2010 \mathrm{GeV}$.
\end{abstract}

DOI: 10.1103/PhysRevLett.120.241801

Primary motivations for building the CERN LHC [1] were to determine the source of electroweak symmetry breaking and to search for physics beyond the standard model (SM). In 2012, the first goal was achieved with the discovery of the Higgs boson $H$ by the ATLAS and CMS Collaborations [24]. In this Letter, we exploit that discovery in a search for events containing high-momentum Higgs bosons in conjunction with hadronic jets and missing momentum transverse to the beam, $\vec{p}_{T}^{\text {miss }}$. Large $p_{T}^{\text {miss }} \equiv\left|\vec{p}_{T}^{\text {miss }}\right|$ can arise from the production of energetic weakly interacting particles that escape detection. A new particle of this type would be a candidate for weakly interacting massive particle (WIMP) dark matter [5-7]. High-momentum Higgs bosons appear rarely in SM processes, and would provide a unique signature of new physics. Such a signature can arise in a variety of models for physics beyond the SM, including extended electroweak sectors [8,9], extended Higgs sectors [10], and supersymmetry (SUSY) [11,12].

The search presented here is based on $35.9 \mathrm{fb}^{-1}$ of proton-proton $(p p)$ collision data at $\sqrt{s}=13 \mathrm{TeV}$ collected in 2016 by the CMS experiment. High-momentum Higgs bosons are reconstructed in the leading $b \bar{b}$ decay channel in a regime in which the two jets from the hadronization of the $b$ quarks overlap with each other.

\footnotetext{
*Full author list given at the end of the Letter.

Published by the American Physical Society under the terms of the Creative Commons Attribution 4.0 International license. Further distribution of this work must maintain attribution to the author(s) and the published article's title, journal citation, and DOI. Funded by SCOAP ${ }^{3}$.
}

They are identified with a recently developed algorithm [13] that employs substructure techniques to large-radius jets. In previous studies CMS [14,15] and ATLAS [16] have searched for signatures with Higgs bosons, jets, and $p_{T}^{\text {miss }}$. This Letter presents the first search for pairs of Lorentz-boosted Higgs bosons produced in association with jets and $p_{T}^{\text {miss }}$.

Supersymmetry [17-24] is a widely studied extension of the SM that posits for each SM particle a new particle, called a superpartner, with a spin that differs from that of its SM counterpart by a half unit. Supersymmetry is attractive as a potential solution to the gauge hierarchy problem [25] that can help to explain the low mass of the Higgs boson without fine tuning of the theory [26-28]. The superpartners of quarks and gluons are squarks $\tilde{q}$ and gluinos $\tilde{g}$, respectively, while neutralinos $\tilde{\chi}^{0}$ and charginos $\tilde{\chi}^{ \pm}$are mixtures of the superpartners of the Higgs and electroweak gauge bosons. In a process such as the simplified model (SMS [29-31]) referred to as $\mathrm{T} 5 \mathrm{HH}$ and illustrated in Fig. 1, gluinos are pair produced and decay into a quark, antiquark, and $\tilde{\chi}_{2}^{0}$, where $\tilde{\chi}_{2}^{0}$ is the second-lightest neutralino. The $\tilde{\chi}_{2}^{0}$ decays into a Higgs boson and the lightest neutralino, $\tilde{\chi}_{1}^{0}$, which we take to be the lightest SUSY particle (LSP) and represents the dark matter candidate. The results of this search are interpreted in the context of this model and the alternate $\mathrm{T} 5 \mathrm{HZ}$, in which the $\tilde{\chi}_{2}^{0}$ branching fractions to $H \tilde{\chi}_{1}^{0}$ and $Z \tilde{\chi}_{1}^{0}$ are both $50 \%$, with primary focus on the T5HH model. We further assume a small $\tilde{g}-\tilde{\chi}_{2}^{0}$ mass splitting and a light $\tilde{\chi}_{1}^{0}$, leading to events with energetic Higgs bosons, large $p_{T}^{\text {miss }}$, and soft quark jets. 


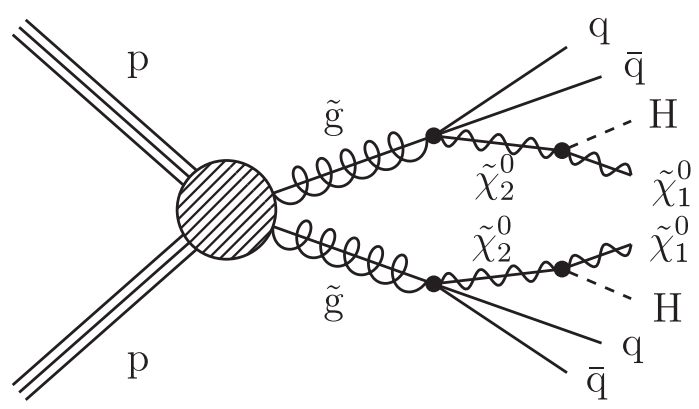

FIG. 1. Diagram for production of Higgs bosons via gluino pair production. We also consider channels in which a $Z$ boson is substituted for $H$ in one of the gluino decays.

A detailed description of the CMS detector, along with a definition of the coordinate system and pertinent kinematical variables, is given in Ref. [32]. Briefly, a cylindrical superconducting solenoid with an inner diameter of $6 \mathrm{~m}$ provides a $3.8 \mathrm{~T}$ axial magnetic field. Within the solenoid volume are a silicon pixel and strip tracker, a lead tungstate crystal electromagnetic calorimeter (ECAL), and a brass and scintillator hadron calorimeter (HCAL). The tracking detectors cover the pseudorapidity range $|\eta|<2.5$. The ECAL and HCAL, each composed of a barrel and two endcap sections, cover $|\eta|<3$.0. Forward calorimeters extend the coverage to $|\eta|<5$.0. Muons are measured within $|\eta|<2.4$ by gas-ionization detectors embedded in the steel flux-return yoke outside the solenoid. The detector is nearly hermetic, permitting accurate measurement of $p_{T}^{\text {miss }}$.

Individual particles are reconstructed with the CMS particle-flow (PF) algorithm [33], which identifies them as photons, charged hadrons, neutral hadrons, electrons, or muons. Jets are defined by forming clusters of PF particles using the anti- $k_{T}$ jet algorithm [34,35] with a distance parameter of 0.8 (AK8) and 0.4 (AK4). The jet energies are corrected for the nonlinear response of the detector [36] and to account for the expected contributions of neutral particles from $p p$ interactions other than the one of interest (pileup) [37]. The quantity $\vec{p}_{\mathrm{T}}^{\text {miss }}$ is reconstructed as the negative of the vector transverse momentum sum over all PF particles, while $H_{T}$ is the sum over AK4 jets of the magnitudes of their transverse momenta, $p_{T}$. The jets for this summation are required to be within the tracker volume and to have a minimum $p_{T}$ of $30 \mathrm{GeV}$ to suppress contributions from pileup.

The lepton content of events is used to characterize signal and control samples. We impose isolation requirements on electron and muon candidates to suppress those arising from jets erroneously identified as leptons, as well as genuine leptons from hadron decays. The isolation criterion is based on the variable $I$, defined as the activity within a cone of radius $\sqrt{(\Delta \phi)^{2}+(\Delta \eta)^{2}}$ around the lepton direction divided by the lepton $p_{T}$. Here activity is defined as the scalar $p_{T}$ sum of charged hadron, neutral hadron, and photon PF particles, corrected for the contributions from pileup. The radius of the cone is 0.2 for lepton $p_{T}<50 \mathrm{GeV}, 10 \mathrm{GeV} / p_{T}$ for $50 \leq p_{T} \leq 200 \mathrm{GeV}$, and 0.05 for $p_{T}>200 \mathrm{GeV}$. The isolation requirement is $I<$ $0.1(0.2)$ for electrons (muons).

To recover electrons or muons that fail tight identification requirements, and $\tau$ leptons via their one-prong hadronic decays, we also make use of isolated charged tracks. For these candidates we require that the scalar $p_{T}$ sum of all other charged-particle tracks within a cone of radius 0.3 around the candidate track direction, divided by the track $p_{T}$, be less than 0.2 if the track is identified as a PF electron or muon and less than 0.1 otherwise. Isolated tracks are required to satisfy $|\eta|<2.4$.

Candidates for $H \rightarrow b \bar{b}$ jets are identified with a heavyflavor tagging algorithm designed to identify a pair of $b$ quarks clustered into a single AK8 jet [13]. The algorithm resolves the decay chains of the two $b$ hadrons and associates secondary vertices along the decay directions, and then computes the likelihood that a jet contains two $b$ hadrons. The jet pruning algorithm described in Ref. [38] is used to improve the jet mass resolution for $H \rightarrow b \bar{b}$ candidates.

The selection of events for analysis begins with the trigger described in Ref. [39]. For this analysis, signal event candidates were recorded by requiring $p_{T}^{\text {miss }}$ and the magnitude $H_{T}^{\text {miss }}$ of the vector $p_{T}$ sum of jets, both computed at the trigger level, to exceed thresholds that varied between 100 and $120 \mathrm{GeV}$ depending on the LHC instantaneous luminosity. The efficiency of this trigger, which exceeds $98 \%$ for events satisfying the selection criteria described below, is measured in data and is taken into account in the analysis. Additional triggers, requiring the presence of charged leptons, photons, or minimum values of $H_{T}$, are used to select samples, described below, employed in the evaluation of backgrounds.

Candidates for signal events are characterized by jets of large angular radius containing a pair of $b$ quarks from the decays of Lorentz-boosted Higgs bosons, accompanied by $p_{T}^{\text {miss }}$ from escaping LSPs. They are required to have no isolated leptons, but we impose no requirements on the number of additional jets in the event. The specific requirements that define the search sample are $p_{T}^{\text {miss }}>300 \mathrm{GeV}$, $H_{T}>600 \mathrm{GeV}$, and at least two AK8 jets with $p_{\mathrm{T}}>$ $300 \mathrm{GeV}$ and mass $m_{J}$ between 50 and $250 \mathrm{GeV}$. We exclude events with either a muon or an electron with $p_{\mathrm{T}}>10 \mathrm{GeV}$, or an isolated track with $m_{T}<100 \mathrm{GeV}$ and $p_{\mathrm{T}}>10(5) \mathrm{GeV}$ for hadronic (leptonic) tracks. Here $m_{T}$ is the transverse mass [40] evaluated from the $\vec{p}_{T}^{\text {miss }}$ and isolated-track $p_{T}$ vectors. The isolated track requirement serves to improve the efficiency for suppressing background from leptonic $W$ decays. To suppress events containing apparent $p_{T}^{\text {miss }}$ caused by mismeasurement of the jet energies, we further impose thresholds on the azimuthal angles between the $\vec{p}_{T}^{\text {miss }}$ vector and those of the (up to) four leading- $p_{T}$ AK4 jets, $\Delta \phi_{1,2,3,4}>0.5,0.5$, 
$0.3,0.3$. For enhanced sensitivity to diverse signal models, events are categorized into three ranges of $p_{T}^{\text {miss }}: 300-500$, $500-700$, and $>700 \mathrm{GeV}$.

Considering the two leading- $p_{T}$ AK8 jets, events are categorized as $0 \mathrm{H}, 1 \mathrm{H}$, or $2 \mathrm{H}$ according to the number of these jets that have a double- $b$ discriminator value greater than 0.3 ( $H$-tagged jets). For true Higgs boson decays the efficiency of this requirement is $50 \%-80 \%$ per AK8 jet depending on the jet $p_{T}$, with the maximum around $500 \mathrm{GeV}$, dropping off to the lower value around $2 \mathrm{TeV}$. Jets are further categorized by $m_{J}$, with the Higgs signal region encompassing the range $85-135 \mathrm{GeV}$, for which the efficiency per jet is $\sim 80 \%$. The remaining mass regions, 50-85 and 135-250 GeV, serve as sidebands. The signal region $A_{1}\left(A_{2}\right)$ is defined as the class of $1 H(2 H)$ events in which both jets lie within the signal mass window. Distributions of $m_{J}$ for the leading- $p_{T}$ jet in $1 H$ and $2 H$ events are shown in Fig. 2, for the observed and simulated events in which the subleading AK8 jet lies within the signal mass window. Here the yields from simulation are scaled to the prediction based on control samples in data, described below, in this mass window. For the T5HH SUSY model the efficiency for selection of events in the signal regions is $9 \%-15 \%$ for $m(\tilde{g})>1200 \mathrm{GeV}$, increasing with $m(\tilde{g})$.

The $m_{J}$ resolution does not permit clean separation of the $H$ and $Z$ boson peaks. The chosen signal window optimizes the selection of $H$ bosons in the absence of $Z$. As noted

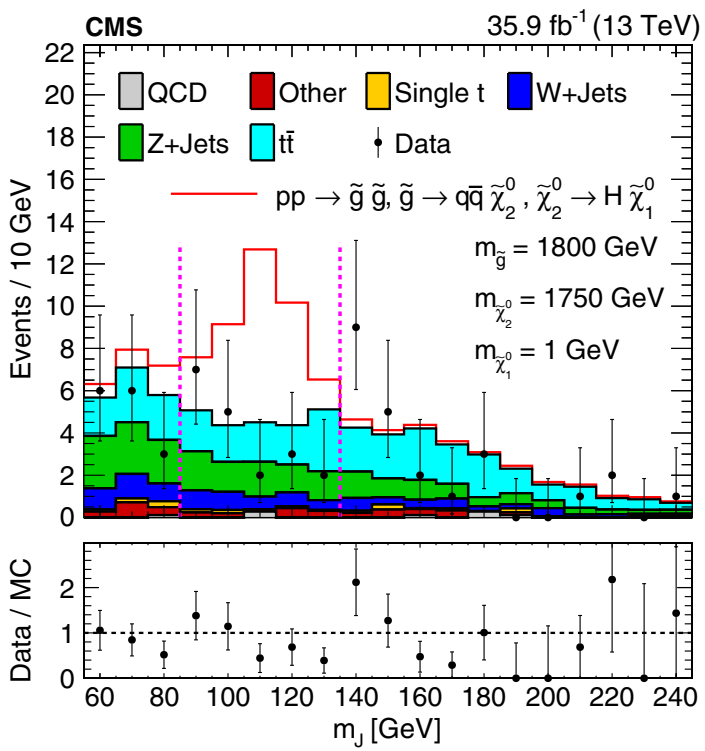

FIG. 2. Observed and expected distributions of the leading- $p_{T}$ jet mass for selected $1 H$ and $2 H$ events with $p_{T}^{\text {miss }}>300 \mathrm{GeV}$. The subleading jet is required to have $m_{J}$ within the signal window denoted by vertical dashed magenta lines. The yields from simulation are scaled to the prediction based on control samples in data, in the signal mass window. A representative signal is shown stacked on top of the backgrounds. The bottom panel shows the ratio of the observed to SM-expected yields. previously we treat both processes as potential signal, and the likelihood fit described below accounts for any signal population in the control regions.

Simulated event samples for SM background processes are used to determine correction factors, typically near unity, that are used in conjunction with observed event yields in control regions to determine the SM background contribution in the signal regions. The production of $t \bar{t}$, $W, Z$, and quantum chromodynamics (QCD) multijet events is simulated with the Monte Carlo (MC) generator MADGRAPH 5_aMC@NLO 2.2.2 [41], with parton distribution functions (PDFs) taken from NNPDF 3.0 [42]. A detailed description of the simulated SM background samples is given in Ref. [43]. The detector simulation is performed with GEANT4 [44]. Simulated event samples for SUSY signal models, used to determine the selection efficiency for signal events, are generated with MADGRAPH 5_aMC@NLO with up to two additional partons at leading order accuracy; they are normalized to cross sections computed to next-to-leading order (NLO) plus next-to-leading logarithmic (NLL) accuracy, based on Ref. [45].

The signal efficiencies from simulation are corrected for the modeling of initial-state radiation as measured in a data control sample [43], the double- $b$ tagging efficiency [13], and the $m_{J}$ resolution observed in data. Systematic uncertainties associated with these corrections are taken into account, as well as those arising from the determination of luminosity, trigger efficiency, PDFs, jet energy scale and resolution, isolated track veto efficiency, renormalization and factorization scales [46,47], and predicted yields from simulation due to limited sample sizes. The largest uncertainties are associated with the modeling of the double- $b$ tagging efficiency $(6 \%)$ and the mass resolution $(1 \%-15 \%)$.

Dominant SM backgrounds arise from events containing jets misidentified as Higgs bosons in conjunction with $W$ or $Z$ bosons, which may originate from top quark decays, that decay to final states with neutrinos, yielding large $p_{T}^{\text {miss }}$. Multijet events in which jets are undermeasured can also give large $p_{T}^{\text {miss; }}$, these backgrounds are highly suppressed by the Higgs boson identification requirements. All backgrounds are estimated from control regions in the data.

The SM backgrounds are estimated by simultaneously extrapolating yields from the $0 \mathrm{H}$ to the $1 \mathrm{H}$ and $2 \mathrm{H} \mathrm{H}$-tag multiplicity regions, and from the $m_{J}$ sideband to the signal window. Events are assigned to the $m_{J}$ sideband if one or both of the leading- $p_{T}$ jets lie outside the signal window. Altogether we define four control regions: $1 \mathrm{H}$ and $2 \mathrm{H}$ events in the $m_{J}$ sidebands, denoted $B_{1}$ and $B_{2}$, respectively; $0 H$ events in the $m_{J}$ signal window, denoted $C$; and $0 H$ events in the $m_{J}$ sidebands, denoted $D$. Each control region is split into three $p_{T}^{\text {miss }}$ bins, corresponding to those defined for the signal regions. Based on the observed yields in these regions within the search sample, the total background is estimated as 


$$
\mathcal{A}_{1,2}=N\left(B_{1,2}\right) \frac{N(C)}{N(D)} \kappa_{1,2},
$$

where the subscript indicates the number of double- $b$ tagged jets, $\mathcal{A}_{1,2}$ is the predicted yield in the $A_{1,2}$ signal region, $N$ is the population of the indicated control region, and $\kappa_{1,2}$ is a correction factor used to account for any correlations between the $H$-tag and $m_{J}$ variables. While $B_{1,2}, C$, and $D$ yields are taken directly from data, $\kappa_{1,2}$ is computed from simulation, corrected for observed discrepancies between data and simulation.

To obtain the corrections to $\kappa_{1,2}$ we compare data with simulation in auxiliary samples, defined to be orthogonal to the search sample, that are enriched in the SM backgrounds expected in the signal region: a single-lepton sample dominated by top quark and $W$ boson production, a sample of single-photon plus jets events serving as proxy for invisibly decaying $Z$ bosons [43], and a sample selected by inverting the $\Delta \phi$ requirement that contains predominantly QCD multijet events. The auxiliary samples satisfy the same requirement in $H_{T}$ and contain the same control and signal regions, $\left(B_{1,2}, C, D\right)$ and $A_{1,2}$, as the search sample. Scale factors given by ratios of the yields in data divided by those from simulation in these auxiliary samples, typically ranging in value from 0.5 to 2.0 , are then applied to the yields of each of the simulated SM backgrounds, before they are combined to obtain the total background yields in the signal and control regions of the search sample. The yields from corrected simulation are found to be statistically compatible with the data in the control regions. From these corrected MC yields we compute $\kappa_{1,2}$ via Eq. (1), for each $p_{T}^{\text {miss }}$ bin; the values are given in Table I below.

Systematic uncertainties in the background prediction enter through the factors $\kappa_{1,2}$. These include contributions from the uncertainties in the relative populations of the SM background processes, the yield statistics and simulation self-consistency in the auxiliary samples, the $p_{T}^{\text {miss }}$ dependence of the scale factors where $p_{T}^{\text {miss }}$ regions are combined to reduce statistical uncertainties, and the self-consistency of the method as applied to the simulated data.

The values of the $\kappa$ factors with their uncertainties for each of the signal regions appear in Table I, along with the

TABLE I. Correction factors, predicted SM background yields, and observed yields, for the signal regions $A_{N_{H}}$. The uncertainties in the predictions include both statistical and systematic contributions.

\begin{tabular}{lcccc}
\hline \hline$N_{H}$ & $p_{T}^{\text {miss }}(\mathrm{GeV})$ & $\kappa$ & Predicted & Observed \\
\hline 1 & $300-500$ & $0.98 \pm 0.11$ & $17.7 \pm 3.8$ & 15 \\
1 & $500-700$ & $0.86 \pm 0.16$ & $3.4 \pm 1.5$ & 2 \\
1 & $>700$ & $0.86 \pm 0.17$ & $0.61 \pm 0.45$ & 1 \\
2 & $300-500$ & $0.73 \pm 0.14$ & $1.52 \pm 0.57$ & 1 \\
2 & $500-700$ & $0.43 \pm 0.12$ & $0.09 \pm 0.08$ & 0 \\
2 & $>700$ & $0.62 \pm 0.30$ & $0.09_{-0.09}^{+0.11}$ & 0 \\
\hline \hline
\end{tabular}

final background yield predictions, and the yields observed in the data. The observations are statistically compatible with those expected from the SM backgrounds, and thus we find no evidence for processes outside the SM.

We compute upper limits on the gluino pair-production cross section using a maximum-likelihood fit in which the free parameters are the signal strength $\mu$, the Poisson means of the total expected yields from SM backgrounds in each of the $B_{1,2}, C$, and $D$ regions, and $\kappa_{1,2}$. The $\kappa_{1,2}$ parameters are constrained with a Gaussian prior to the expected values, with their statistical and systematic uncertainties. The signal model in the fit accounts for the populations of control as well as signal regions. Additional nuisance parameters account for systematic uncertainties in the yields predicted by the signal model.

We evaluate 95\% confidence level (CL) upper limits based on the asymptotic form of a likelihood ratio test statistic [48], in conjunction with the $\mathrm{CL}_{S}$ criterion described in Refs. [49-51]. The test statistic is $q(\mu)=$ $-2 \ln \left(\mathcal{L}_{\mu} / \mathcal{L}_{\text {max }}\right)$, where $\mathcal{L}_{\max }$ is the maximum likelihood determined by allowing all parameters, including $\mu$, to vary, and $\mathcal{L}_{\mu}$ is the maximum likelihood for fixed $\mu$. Expected and observed 95\% CL upper limits, and the predicted gluino pair-production cross sections, are shown in Fig. 3 for two choices of the $\tilde{\chi}_{2}^{0}$ decay branching fractions, taking $m\left(\tilde{\chi}_{1}^{0}\right)=$ $1 \mathrm{GeV}$ and $m(\tilde{g})-m\left(\tilde{\chi}_{2}^{0}\right)=50 \mathrm{GeV}$. That is, we choose a model with a light LSP and a compressed spectrum for the heavy SUSY particles, thereby ensuring a Lorentz-boosted topology.

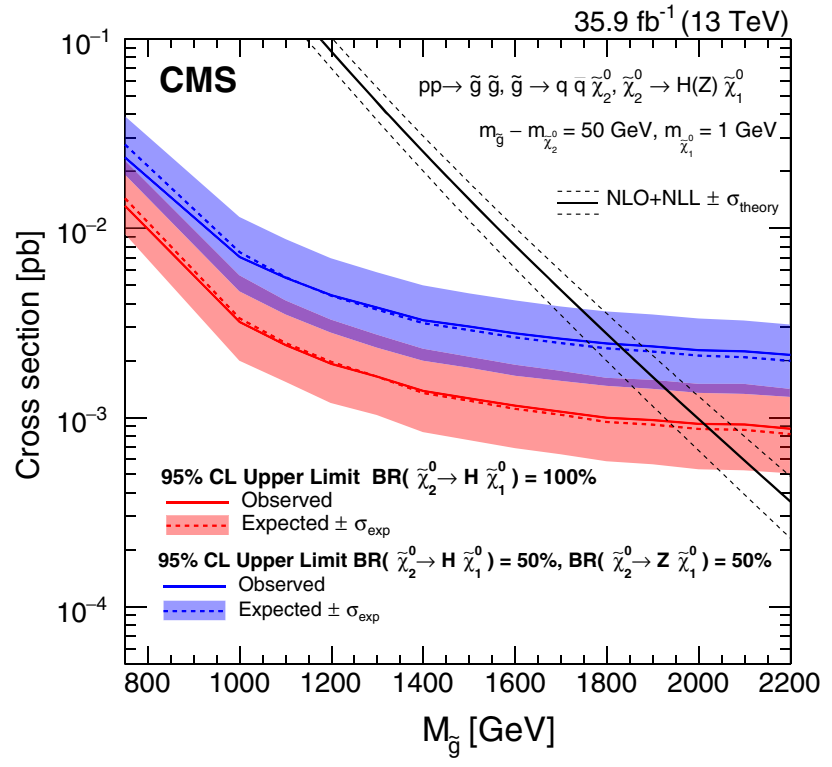

FIG. 3. Observed and expected cross section upper bounds at 95\% CL for the T5HH and T5HZ models. The solid and dashed black lines show the SMS gluino-gluino production cross section with its uncertainty. The solid red (blue) line shows the observed limit for the T5HH (T5HZ) model; for each the like-colored dashed line and shaded band show the expected limit and the range associated with the experimental uncertainties. 
In summary, this Letter has presented a search for production of energetic Higgs bosons in conjunction with large missing transverse momentum in proton-proton collisions. Higgs bosons with transverse momentum in the range $300 \mathrm{GeV}$ to about $2 \mathrm{TeV}$ are reconstructed as wide-cone jets with substructure indicative of the decay of the Higgs boson to a pair of $b$ quarks. Background from standard model processes is estimated from data control regions. The observed event yields are found to be statistically compatible with these backgrounds.

The results are broadly applicable to models leading to signatures with energetic Higgs bosons and missing momentum. Here they are interpreted in the context of a simplified model of supersymmetry in which gluinos are pair produced and subsequently decay into several quarks, a Higgs or $Z$ boson, and the lightest supersymmetric particle, a neutralino $\tilde{\chi}_{1}^{0}$. Gluinos with masses below 2010 (1825) $\mathrm{GeV}$ are excluded under the assumption of a large mass splitting between the next-to-lightest and lightest supersymmetric particle and that the branching fraction of $\tilde{\chi}_{2}^{0} \rightarrow H \tilde{\chi}_{1}^{0}$ is $100 \%$ (50\%). These are the first limits for pair production of gluinos measured in these decay channels.

We congratulate our colleagues in the CERN accelerator departments for the excellent performance of the LHC and thank the technical and administrative staffs at CERN and at other CMS institutes for their contributions to the success of the CMS effort. In addition, we gratefully acknowledge the computing centers and personnel of the Worldwide LHC Computing Grid for delivering so effectively the computing infrastructure essential to our analyses. Finally, we acknowledge the enduring support for the construction and operation of the LHC and the CMS detector provided by the following funding agencies: BMWFW and FWF (Austria); FNRS and FWO (Belgium); CNPq, CAPES, FAPERJ, and FAPESP (Brazil); MES (Bulgaria); CERN; CAS, MoST, and NSFC (China); COLCIENCIAS (Colombia); MSES and CSF (Croatia); RPF (Cyprus); SENESCYT (Ecuador); MoER, ERC IUT, and ERDF (Estonia); Academy of Finland, MEC, and HIP (Finland); CEA and CNRS/IN2P3 (France); BMBF, DFG, and HGF (Germany); GSRT (Greece); OTKA and NIH (Hungary); DAE and DST (India); IPM (Iran); SFI (Ireland); INFN (Italy); MSIP and NRF (Republic of Korea); LAS (Lithuania); MOE and UM (Malaysia); BUAP, CINVESTAV, CONACYT, LNS, SEP, and UASLP-FAI (Mexico); MBIE (New Zealand); PAEC (Pakistan); MSHE and NSC (Poland); FCT (Portugal); JINR (Dubna); MON, RosAtom, RAS, RFBR and RAEP (Russia); MESTD (Serbia); SEIDI, CPAN, PCTI and FEDER (Spain); Swiss Funding Agencies (Switzerland); MST (Taipei); ThEPCenter, IPST, STAR, and NSTDA (Thailand); TUBITAK and TAEK (Turkey); NASU and SFFR (Ukraine); STFC (United Kingdom); DOE and NSF (USA).
[1] L. Evans and P. Bryant, LHC machine, J. Instrum. 3, S08001 (2008).

[2] ATLAS Collaboration, Observation of a new particle in the search for the standard model Higgs boson with the ATLAS detector at the LHC, Phys. Lett. B 716, 1 (2012).

[3] CMS Collaboration, Observation of a new boson at a mass of $125 \mathrm{GeV}$ with the CMS experiment at the LHC, Phys. Lett. B 716, 30 (2012).

[4] ATLAS and CMS Collaborations, Combined Measurement of the Higgs Boson Mass in $p p$ Collisions at $\sqrt{s}=7$ and $8 \mathrm{TeV}$ with the ATLAS and CMS Experiments, Phys. Rev. Lett. 114, 191803 (2015).

[5] C. Patrignani et al. (Particle Data Group), Review of particle physics, Chin. Phys. C 40, 100001 (2016), section 26.

[6] F. Zwicky, Die rotverschiebung von extragalaktischen nebeln, Helv. Phys. Acta 6, 110 (1933).

[7] V. C. Rubin and W. K. Ford Jr., Rotation of the Andromeda nebula from a spectroscopic survey of emission regions, Astrophys. J. 159, 379 (1970).

[8] B. A. Dobrescu and C. Frugiuele, Hidden GeV-Scale Interactions of Quarks, Phys. Rev. Lett. 113, 061801 (2014).

[9] B. A. Dobrescu, Leptophobic boson signals with leptons, jets and missing energy, arXiv:1506.04435.

[10] R. K. Barman, B. Bhattacherjee, A. Chakraborty, and A. Choudhury, Study of MSSM heavy Higgs bosons decaying into charginos and neutralinos, Phys. Rev. D 94, 075013 (2016).

[11] S. Gori, P. Schwaller, and C. E. M. Wagner, Search for Higgs bosons in supersymmetric cascade decays and neutralino dark matter, Phys. Rev. D 83, 115022 (2011).

[12] Z. Kang, P. Ko, and J. Li, New Physics Opportunities in the Boosted Di-Higgs-Boson Plus Missing Transverse Energy Signature, Phys. Rev. Lett. 116, 131801 (2016).

[13] CMS Collaboration, Identification of heavy-flavour jets with the CMS detector in pp collisions at $13 \mathrm{TeV}$, J. Instrum. 13, P05011 (2018).

[14] CMS Collaboration, Search for Top Squark and Higgsino Production Using Diphoton Higgs Boson Decays, Phys. Rev. Lett. 112, 161802 (2014).

[15] CMS Collaboration, Searches for electroweak neutralino and chargino production in channels with Higgs, Z, and W bosons in pp collisions at $8 \mathrm{TeV}$, Phys. Rev. D 90, 092007 (2014).

[16] ATLAS Collaboration, Search for supersymmetry in events with photons, bottom quarks, and missing transverse momentum in proton-proton collisions at a centre-of-mass energy of $7 \mathrm{TeV}$ with the ATLAS detector, Phys. Lett. B 719, 261 (2013).

[17] P. Ramond, Dual theory for free fermions, Phys. Rev. D 3, 2415 (1971).

[18] Yu. A. Gol'fand and E. P. Likhtman, Extension of the algebra of Poincaré group generators and violation of $\mathrm{P}$ invariance, JETP Lett. 13, 323 (1971).

[19] A. Neveu and J. H. Schwarz, Factorizable dual model of pions, Nucl. Phys. B31, 86 (1971).

[20] D. V. Volkov and V. P. Akulov, Possible universal neutrino interaction, JETP Lett. 16, 438 (1972).

[21] J. Wess and B. Zumino, A Lagrangian model invariant under supergauge transformations, Phys. Lett. B 49, 52 (1974). 
[22] J. Wess and B. Zumino, Supergauge transformations in four dimensions, Nucl. Phys. B70, 39 (1974).

[23] P. Fayet, Supergauge invariant extension of the Higgs mechanism and a model for the electron and its neutrino, Nucl. Phys. B90, 104 (1975).

[24] H. P. Nilles, Supersymmetry, supergravity and particle physics, Phys. Rep. 110, 1 (1984).

[25] R. Barbieri and G. F. Giudice, Upper bounds on supersymmetric particle masses, Nucl. Phys. B306, 63 (1988).

[26] S. Dimopoulos and G. F. Giudice, Naturalness constraints in supersymmetric theories with nonuniversal soft terms, Phys. Lett. B 357, 573 (1995).

[27] R. Barbieri and D. Pappadopulo, S-particles at their naturalness limits, J. High Energy Phys. 10 (2009) 061.

[28] M. Papucci, J. T. Ruderman, and A. Weiler, Natural SUSY endures, J. High Energy Phys. 09 (2012) 035.

[29] J. Alwall, P. C. Schuster, and N. Toro, Simplified models for a first characterization of new physics at the LHC, Phys. Rev. D 79, 075020 (2009).

[30] J. Alwall, M.-P. Le, M. Lisanti, and J. G. Wacker, Modelindependent jets plus missing energy searches, Phys. Rev. D 79, 015005 (2009).

[31] D. Alves et al., Simplified models for LHC new physics searches, J. Phys. G 39, 105005 (2012).

[32] CMS Collaboration, The CMS experiment at the CERN LHC, J. Instrum. 3, S08004 (2008).

[33] CMS Collaboration, Particle-flow reconstruction and global event description with the CMS detector, J. Instrum. 12, P10003 (2017).

[34] M. Cacciari, G. P. Salam, and G. Soyez, The anti- $k_{t}$ jet clustering algorithm, J. High Energy Phys. 04 (2008) 063.

[35] M. Cacciari, G. P. Salam, and G. Soyez, FastJet user manual, Eur. Phys. J. C 72, 1896 (2012).

[36] CMS Collaboration, Jet energy scale and resolution in the CMS experiment in pp collisions at $8 \mathrm{TeV}$, J. Instrum. 12, P02014 (2017).

[37] M. Cacciari and G. P. Salam, Pileup subtraction using jet areas, Phys. Lett. B 659, 119 (2008).

[38] S. D. Ellis, C. K. Vermilion, and J. R. Walsh, Recombination algorithms and jet substructure: Pruning as a tool for heavy particle searches, Phys. Rev. D 81, 094023 (2010).

[39] CMS Collaboration, The CMS trigger system, J. Instrum. 12, P01020 (2017).

[40] G. Arnison et al. (UA1), Experimental observation of isolated large transverse energy electrons with associated missing energy at $\sqrt{s}=540 \mathrm{GeV}$, Phys. Lett. B 122, 103 (1983).

[41] J. Alwall, R. Frederix, S. Frixione, V. Hirschi, F. Maltoni, O. Mattelaer, H.-S. Shao, T. Stelzer, P. Torrielli, and M. Zaro, The automated computation of tree-level and next-to-leading order differential cross sections, and their matching to parton shower simulations, J. High Energy Phys. 07 (2014) 079 .

[42] R. D. Ball et al. (NNPDF), Parton distributions for the LHC Run II, J. High Energy Phys. 04 (2015) 040.

[43] CMS Collaboration, Search for supersymmetry in multijet events with missing transverse momentum in protonproton collisions at $13 \mathrm{TeV}$, Phys. Rev. D 96, 032003 (2017).

[44] S. Agostinelli et al. (GeAnt4), Geant4-A simulation toolkit, Nucl. Instrum. Methods, Phys. Res., Sect. A 506, 250 (2003).

[45] C. Borschensky, M. Krämer, A. Kulesza, M. Mangano, S. Padhi, T. Plehn, and X. Portell, Squark and gluino production cross sections in pp collisions at $\sqrt{s}=13,14,33$ and 100 TeV, Eur. Phys. J. C 74, 3174 (2014).

[46] S. Catani, D. de Florian, M. Grazzini, and P. Nason, Soft gluon resummation for Higgs boson production at hadron colliders, J. High Energy Phys. 07 (2003) 028.

[47] M. Cacciari, S. Frixione, M. L. Mangano, P. Nason, and G. Ridolfi, The $t \bar{t}$ cross-section at $1.8 \mathrm{TeV}$ and $1.96 \mathrm{TeV}$ : A study of the systematics due to parton densities and scale dependence, J. High Energy Phys. 04 (2004) 068.

[48] G. Cowan, K. Cranmer, E. Gross, and O. Vitells, Asymptotic formulae for likelihood-based tests of new physics, Eur. Phys. J. C 71, 1554 (2011); Erratum, Eur. Phys. J. C 73, 2501 (2013).

[49] A. L. Read, Presentation of search results: The $C L_{s}$ technique, in Durham IPPP Workshop: Advanced Statistical Techniques in Particle Physics (Durham, UK, 2002), p. 2693; J. Phys. G 28, 2693 (2002).

[50] T. Junk, Confidence level computation for combining searches with small statistics, Nucl. Instrum. Methods Phys. Res., Sect. A 434, 435 (1999).

[51] ATLAS and CMS Collaborations, LHC Higgs Combination Group, Procedure for the LHC Higgs boson search combination in Summer 2011, Technical Report Nos. CMS-NOTE-2011-005, ATL-PHYS-PUB-2011-11, CERN, 2011.

A. M. Sirunyan, ${ }^{1}$ A. Tumasyan, ${ }^{1}$ W. Adam, ${ }^{2}$ F. Ambrogi, ${ }^{2}$ E. Asilar, ${ }^{2}$ T. Bergauer, ${ }^{2}$ J. Brandstetter, ${ }^{2}$ E. Brondolin, ${ }^{2}$ M. Dragicevic, ${ }^{2}$ J. Erö, ${ }^{2}$ A. Escalante Del Valle, ${ }^{2}$ M. Flechl, ${ }^{2}$ M. Friedl, ${ }^{2}$ R. Frühwirth, ${ }^{2, b}$ V. M. Ghete, ${ }^{2}$ J. Grossmann, ${ }^{2}$ J. Hrubec, ${ }^{2}$ M. Jeitler, ${ }^{2, b}$ A. König, ${ }^{2}$ N. Krammer, ${ }^{2}$ I. Krätschmer, ${ }^{2}$ D. Liko, ${ }^{2}$ T. Madlener, ${ }^{2}$ I. Mikulec, ${ }^{2}$ E. Pree, ${ }^{2}$ N. Rad, ${ }^{2}$ H. Rohringer, ${ }^{2}$ J. Schieck, ${ }^{2, b}$ R. Schöfbeck, ${ }^{2}$ M. Spanring, ${ }^{2}$ D. Spitzbart, ${ }^{2}$ A. Taurok, ${ }^{2}$ W. Waltenberger, ${ }^{2}$ J. Wittmann, ${ }^{2}$ C.-E. Wulz ${ }^{2, b}$ M. Zarucki, ${ }^{2}$ V. Chekhovsky, ${ }^{3}$ V. Mossolov, ${ }^{3}$ J. Suarez Gonzalez, ${ }^{3}$ E. A. De Wolf, ${ }^{4}$ D. Di Croce, ${ }^{4}$ X. Janssen, ${ }^{4}$ J. Lauwers, ${ }^{4}$ M. Pieters, ${ }^{4}$ M. Van De Klundert, ${ }^{4}$ H. Van Haevermaet, ${ }^{4}$ P. Van Mechelen, ${ }^{4}$ N. Van Remortel, ${ }^{4}$ S. Abu Zeid, ${ }^{5}$ F. Blekman, ${ }^{5}$ J. D’Hondt, ${ }^{5}$ I. De Bruyn, ${ }^{5}$ J. De Clercq, ${ }^{5}$ K. Deroover, ${ }^{5}$ G. Flouris, ${ }^{5}$ D. Lontkovskyi, ${ }^{5}$ S. Lowette, ${ }^{5}$ I. Marchesini ${ }^{5}$ S. Moortgat, ${ }^{5}$ L. Moreels, ${ }^{5}$ Q. Python, ${ }^{5}$ K. Skovpen, ${ }^{5}$ S. Tavernier, ${ }^{5}$ W. Van Doninck, ${ }^{5}$ P. Van Mulders, ${ }^{5}$ I. Van Parijs, ${ }^{5}$ D. Beghin, ${ }^{6}$ B. Bilin, ${ }^{6}$ H. Brun, ${ }^{6}$ B. Clerbaux, ${ }^{6}$ G. De Lentdecker, ${ }^{6}$ H. Delannoy, ${ }^{6}$ B. Dorney, ${ }^{6}$ G. Fasanella, ${ }^{6}$ 
L. Favart, ${ }^{6}$ R. Goldouzian, ${ }^{6}$ A. Grebenyuk, ${ }^{6}$ A. K. Kalsi, ${ }^{6}$ T. Lenzi, ${ }^{6}$ J. Luetic, ${ }^{6}$ T. Seva, ${ }^{6}$ E. Starling, ${ }^{6}$ C. Vander Velde, ${ }^{6}$ P. Vanlaer, ${ }^{6}$ D. Vannerom, ${ }^{6}$ R. Yonamine, ${ }^{6}$ T. Cornelis, ${ }^{7}$ D. Dobur, ${ }^{7}$ A. Fagot, ${ }^{7}$ M. Gul, ${ }^{7}$ I. Khvastunov, ${ }^{7, c}$ D. Poyraz, ${ }^{7}$ C. Roskas, ${ }^{7}$ D. Trocino, ${ }^{7}$ M. Tytgat, ${ }^{7}$ W. Verbeke, ${ }^{7}$ M. Vit, ${ }^{7}$ N. Zaganidis, ${ }^{7}$ H. Bakhshiansohi, ${ }^{8}$ O. Bondu, ${ }^{8}$ S. Brochet, ${ }^{8}$ G. Bruno, ${ }^{8}$ C. Caputo, ${ }^{8}$ A. Caudron,${ }^{8}$ P. David,${ }^{8}$ S. De Visscher, ${ }^{8}$ C. Delaere, ${ }^{8}$ M. Delcourt, ${ }^{8}$ B. Francois,${ }^{8}$ A. Giammanco, ${ }^{8}$ G. Krintiras, ${ }^{8}$ V. Lemaitre, ${ }^{8}$ A. Magitteri, ${ }^{8}$ A. Mertens, ${ }^{8}$ M. Musich, ${ }^{8}$ K. Piotrzkowski, ${ }^{8}$ L. Quertenmont, ${ }^{8}$ A. Saggio, ${ }^{8}$ M. Vidal Marono, ${ }^{8}$ S. Wertz, ${ }^{8}$ J. Zobec, ${ }^{8}$ W. L. Aldá Júnior, ${ }^{9}$ F. L. Alves, ${ }^{9}$ G. A. Alves, ${ }^{9}$ L. Brito, ${ }^{9}$ G. Correia Silva, ${ }^{9}$ C. Hensel,,${ }^{9}$ A. Moraes, ${ }^{9}$ M. E. Pol, ${ }^{9}$ P. Rebello Teles, ${ }^{9}$ E. Belchior Batista Das Chagas, ${ }^{10}$ W. Carvalho, ${ }^{10}$ J. Chinellato, ${ }^{10, d}$ E. Coelho, ${ }^{10}$ E. M. Da Costa, ${ }^{10}$ G. G. Da Silveira, ${ }^{10, e}$ D. De Jesus Damiao ${ }^{10}$ S. Fonseca De Souza, ${ }^{10}$

L. M. Huertas Guativa, ${ }^{10}$ H. Malbouisson, ${ }^{10}$ M. Medina Jaime, ${ }^{10, f}$ M. Melo De Almeida, ${ }^{10}$ C. Mora Herrera,${ }^{10}$ L. Mundim, ${ }^{10}$ H. Nogima,${ }^{10}$ L. J. Sanchez Rosas, ${ }^{10}$ A. Santoro, ${ }^{10}$ A. Sznajder, ${ }^{10}$ M. Thiel,${ }^{10}$ E. J. Tonelli Manganote, ${ }^{10, d}$

F. Torres Da Silva De Araujo, ${ }^{10}$ A. Vilela Pereira, ${ }^{10}$ S. Ahuja, ${ }^{11 a}$ C. A. Bernardes, ${ }^{11 a}$ T. R. Fernandez Perez Tomei, ${ }^{11 a}$ E. M. Gregores, ${ }^{11 \mathrm{~b}}$ P. G. Mercadante, ${ }^{11 \mathrm{~b}}$ S. F. Novaes, ${ }^{11 \mathrm{a}}$ Sandra S. Padula, ${ }^{11 \mathrm{a}}$ D. Romero Abad, ${ }^{11 \mathrm{~b}}$ J. C. Ruiz Vargas, ${ }^{11 \mathrm{a}}$ A. Aleksandrov, ${ }^{12}$ R. Hadjiiska, ${ }^{12}$ P. Iaydjiev, ${ }^{12}$ A. Marinov, ${ }^{12}$ M. Misheva, ${ }^{12}$ M. Rodozov, ${ }^{12}$ M. Shopova, ${ }^{12}$ G. Sultanov, ${ }^{12}$ A. Dimitrov, ${ }^{13}$ L. Litov, ${ }^{13}$ B. Pavlov, ${ }^{13}$ P. Petkov, ${ }^{13}$ W. Fang, ${ }^{14, g}$ X. Gao, ${ }^{14, g}$ L. Yuan, ${ }^{14}$ M. Ahmad, ${ }^{15}$ J. G. Bian, ${ }^{15}$ G. M. Chen, ${ }^{15}$ H. S. Chen, ${ }^{15}$ M. Chen, ${ }^{15}$ Y. Chen, ${ }^{15}$ C. H. Jiang, ${ }^{15}$ D. Leggat,${ }^{15}$ H. Liao, ${ }^{15}$ Z. Liu, ${ }^{15}$ F. Romeo, ${ }^{15}$ S. M. Shaheen, ${ }^{15}$ A. Spiezia, ${ }^{15}$ J. Tao,${ }^{15}$ C. Wang, ${ }^{15}$ Z. Wang, ${ }^{15}$ E. Yazgan,${ }^{15}$ H. Zhang, ${ }^{15}$ J. Zhao, ${ }^{15}$ Y. Ban, ${ }^{16}$ G. Chen, ${ }^{16}$ J. Li, ${ }^{16}$ Q. Li ${ }^{16}$ S. Liu, ${ }^{16}$ Y. Mao, ${ }^{16}$ S. J. Qian, ${ }^{16}$ D. Wang, ${ }^{16}$ Z. Xu, ${ }^{16}$ Y. Wang, ${ }^{17}$ C. Avila, ${ }^{18}$ A. Cabrera,${ }^{18}$ C. A. Carrillo Montoya, ${ }^{18}$ L. F. Chaparro Sierra ${ }^{18}$ C. Florez, ${ }^{18}$ C. F. González Hernández, ${ }^{18}$ J. D. Ruiz Alvarez, ${ }^{18}$ M. A. Segura Delgado, ${ }^{18}$ B. Courbon, ${ }^{19}$ N. Godinovic, ${ }^{19}$ D. Lelas, ${ }^{19}$ I. Puljak, ${ }^{19}$ P. M. Ribeiro Cipriano, ${ }^{19}$ T. Sculac, ${ }^{19}$ Z. Antunovic, ${ }^{20}$ M. Kovac, ${ }^{20}$ V. Brigljevic, ${ }^{21}$ D. Ferencek, ${ }^{21}$ K. Kadija, ${ }^{21}$ B. Mesic, ${ }^{21}$ A. Starodumov, ${ }^{21, h}$ T. Susa, ${ }^{21}$ M. W. Ather, ${ }^{22}$ A. Attikis, ${ }^{22}$ G. Mavromanolakis, ${ }^{22}$ J. Mousa, ${ }^{22}$ C. Nicolaou, ${ }^{22}$ F. Ptochos, ${ }^{22}$ P. A. Razis, ${ }^{22}$ H. Rykaczewski, ${ }^{22}$ M. Finger, ${ }^{23, \mathrm{i}}$ M. Finger Jr. ${ }^{23, \mathrm{i}}$ E. Carrera Jarrin, ${ }^{24}$ A. A. Abdelalim, ${ }^{25, \mathrm{j}, \mathrm{k}}$ S. Elgammal, ${ }^{25,1}$ S. Khalil, ${ }^{25, \mathrm{k}}$ S. Bhowmik, ${ }^{26}$ R. K. Dewanjee, ${ }^{26}$ M. Kadastik,${ }^{26}$ L. Perrini, ${ }^{26}$ M. Raidal, ${ }^{26}$ C. Veelken, ${ }^{26}$ P. Eerola, ${ }^{27}$ H. Kirschenmann, ${ }^{27}$ J. Pekkanen, ${ }^{27}$ M. Voutilainen, ${ }^{27}$ J. Havukainen, ${ }^{28}$ J. K. Heikkilä, ${ }^{28}$ T. Järvinen, ${ }^{28}$ V. Karimäki, ${ }^{28}$ R. Kinnunen, ${ }^{28}$ T. Lampén, ${ }^{28}$ K. Lassila-Perini, ${ }^{28}$ S. Laurila, ${ }^{28}$ S. Lehti, ${ }^{28}$ T. Lindén, ${ }^{28}$ P. Luukka, ${ }^{28}$ T. Mäenpää, ${ }^{28}$ H. Siikonen, ${ }^{28}$ E. Tuominen, ${ }^{28}$ J. Tuominiemi, ${ }^{28}$ T. Tuuva, ${ }^{29}$ M. Besancon, ${ }^{30}$ F. Couderc,${ }^{30}$ M. Dejardin, ${ }^{30}$ D. Denegri, ${ }^{30}$ J. L. Faure, ${ }^{30}$ F. Ferri, ${ }^{30}$ S. Ganjour, ${ }^{30}$ S. Ghosh, ${ }^{30}$ A. Givernaud,${ }^{30}$ P. Gras,${ }^{30}$ G. Hamel de Monchenault,${ }^{30}$ P. Jarry, ${ }^{30}$ C. Leloup,${ }^{30}$

E. Locci, ${ }^{30}$ M. Machet,${ }^{30}$ J. Malcles,${ }^{30}$ G. Negro, ${ }^{30}$ J. Rander, ${ }^{30}$ A. Rosowsky, ${ }^{30}$ M. Ö. Sahin, ${ }^{30}$ M. Titov, ${ }^{30}$

A. Abdulsalam, ${ }^{31, \mathrm{~m}}$ C. Amendola, ${ }^{31}$ I. Antropov, ${ }^{31}$ S. Baffioni, ${ }^{31}$ F. Beaudette,${ }^{31}$ P. Busson,${ }^{31}$ L. Cadamuro, ${ }^{31}$ C. Charlot, ${ }^{31}$ R. Granier de Cassagnac, ${ }^{31}$ M. Jo, ${ }^{31}$ I. Kucher, ${ }^{31}$ S. Lisniak, ${ }^{31}$ A. Lobanov, ${ }^{31}$ J. Martin Blanco, ${ }^{31}$ M. Nguyen, ${ }^{31}$ C. Ochando, ${ }^{31}$ G. Ortona,${ }^{31}$ P. Paganini, ${ }^{31}$ P. Pigard, ${ }^{31}$ R. Salerno, ${ }^{31}$ J. B. Sauvan ${ }^{31}$ Y. Sirois, ${ }^{31}$ A. G. Stahl Leiton, ${ }^{31}$ Y. Yilmaz, ${ }^{31}$ A. Zabi, ${ }^{31}$ A. Zghiche, ${ }^{31}$ J.-L. Agram, ${ }^{32, n}$ J. Andrea, ${ }^{32}$ D. Bloch, ${ }^{32}$ J.-M. Brom, ${ }^{32}$ M. Buttignol, ${ }^{32}$ E. C. Chabert ${ }^{32}$ C. Collard, ${ }^{32}$ E. Conte, ${ }^{32, \mathrm{n}}$ X. Coubez, ${ }^{32}$ F. Drouhin, ${ }^{32, \mathrm{n}}$ J.-C. Fontaine, ${ }^{32, \mathrm{n}}$ D. Gelé, ${ }^{32}$ U. Goerlach ${ }^{32}$ M. Jansová, ${ }^{32}$ P. Juillot, ${ }^{32}$ A.-C. Le Bihan, ${ }^{32}$ N. Tonon, ${ }^{32}$ P. Van Hove, ${ }^{32}$ S. Gadrat,${ }^{33}$ S. Beauceron, ${ }^{34}$ C. Bernet,${ }^{34}$ G. Boudoul, ${ }^{34}$ N. Chanon, ${ }^{34}$ R. Chierici, ${ }^{34}$ D. Contardo, ${ }^{34}$ P. Depasse, ${ }^{34}$ H. El Mamouni, ${ }^{34}$ J. Fay, ${ }^{34}$ L. Finco, ${ }^{34}$ S. Gascon,${ }^{34}$ M. Gouzevitch, ${ }^{34}$ G. Grenier, ${ }^{34}$ B. Ille, ${ }^{34}$ F. Lagarde, ${ }^{34}$ I. B. Laktineh, ${ }^{34}$ H. Lattaud, ${ }^{34}$ M. Lethuillier, ${ }^{34}$ L. Mirabito, ${ }^{34}$ A. L. Pequegnot, ${ }^{34}$ S. Perries, ${ }^{34}$ A. Popov, ${ }^{34,0}$ V. Sordini, ${ }^{34}$ M. Vander Donckt, ${ }^{34}$ S. Viret, ${ }^{34}$ S. Zhang, ${ }^{34}$ A. Khvedelidze,${ }^{35, i}$ L. Rurua,${ }^{36}$ C. Autermann, ${ }^{37}$ L. Feld, ${ }^{37}$ M. K. Kiesel, ${ }^{37}$ K. Klein, ${ }^{37}$ M. Lipinski, ${ }^{37}$ M. Preuten, ${ }^{37}$ C. Schomakers,${ }^{37}$ J. Schulz, ${ }^{37}$ M. Teroerde,${ }^{37}$ B. Wittmer, ${ }^{37}$ V. Zhukov, ${ }^{37,0}$ A. Albert, ${ }^{38}$ D. Duchardt, ${ }^{38}$ M. Endres,${ }^{38}$ M. Erdmann, ${ }^{38}$ S. Erdweg, ${ }^{38}$ T. Esch, ${ }^{38}$ R. Fischer, ${ }^{38}$ A. Güth, ${ }^{38}$ T. Hebbeker, ${ }^{38}$ C. Heidemann, ${ }^{38}$ K. Hoepfner, ${ }^{38}$ S. Knutzen, ${ }^{38}$ M. Merschmeyer, ${ }^{38}$ A. Meyer, ${ }^{38}$ P. Millet, ${ }^{38}$ S. Mukherjee, ${ }^{38}$ T. Pook, ${ }^{38}$ M. Radziej, ${ }^{38}$ H. Reithler, ${ }^{38}$ M. Rieger, ${ }^{38}$ F. Scheuch,$^{38}$ D. Teyssier, ${ }^{38}$ S. Thüer,${ }^{38}$ G. Flügge,${ }^{39}$ B. Kargoll, ${ }^{39}$ T. Kress,${ }^{39}$ A. Künsken, ${ }^{39}$ T. Müller, ${ }^{39}$ A. Nehrkorn, ${ }^{39}$ A. Nowack, ${ }^{39}$ C. Pistone, ${ }^{39}$ O. Pooth,${ }^{39}$ A. Stahl,${ }^{39, p}$ M. Aldaya Martin, ${ }^{40}$ T. Arndt, ${ }^{40}$ C. Asawatangtrakuldee, ${ }^{40}$ K. Beernaert, ${ }^{40}$ O. Behnke, ${ }^{40}$ U. Behrens, ${ }^{40}$ A. Bermúdez Martínez,${ }^{40}$ A. A. Bin Anuar, ${ }^{40}$ K. Borras, ${ }^{40, q}$ V. Botta, ${ }^{40}$ A. Campbell, ${ }^{40}$ P. Connor, ${ }^{40}$ C. Contreras-Campana, ${ }^{40}$ F. Costanza, ${ }^{40}$ A. De Wit, ${ }^{40}$ C. Diez Pardos,${ }^{40}$ G. Eckerlin, ${ }^{40}$ D. Eckstein, ${ }^{40}$ T. Eichhorn, ${ }^{40}$ E. Eren,${ }^{40}$ E. Gallo, ${ }^{40, r}$ J. Garay Garcia, ${ }^{40}$ A. Geiser, ${ }^{40}$ J. M. Grados Luyando, ${ }^{40}$ A. Grohsjean, ${ }^{40}$ P. Gunnellini, ${ }^{40}$ M. Guthoff, ${ }^{40}$ A. Harb ${ }^{40}$ J. Hauk,${ }^{40}$ M. Hempel, ${ }^{40, s}$ H. Jung, ${ }^{40}$ M. Kasemann, ${ }^{40}$ J. Keaveney, ${ }^{40}$ C. Kleinwort, ${ }^{40}$ I. Korol, ${ }^{40}$ D. Krücker ${ }^{40}$ W. Lange, ${ }^{40}$ A. Lelek, ${ }^{40}$ T. Lenz,${ }^{40}$ K. Lipka, ${ }^{40}$ W. Lohmann, ${ }^{40, s}$ R. Mankel, ${ }^{40}$ I.-A. Melzer-Pellmann, ${ }^{40}$ A. B. Meyer, ${ }^{40}$ M. Meyer, ${ }^{40}$ M. Missiroli, ${ }^{40}$ G. Mittag, ${ }^{40}$ J. Mnich, ${ }^{40}$ A. Mussgiller, ${ }^{40}$ D. Pitzl, ${ }^{40}$ 
A. Raspereza, ${ }^{40}$ M. Savitskyi ${ }^{40}$ P. Saxena, ${ }^{40}$ R. Shevchenko, ${ }^{40}$ N. Stefaniuk, ${ }^{40}$ H. Tholen, ${ }^{40}$ G. P. Van Onsem, ${ }^{40}$ R. Walsh,${ }^{40}$ Y. Wen, ${ }^{40}$ K. Wichmann, ${ }^{40}$ C. Wissing,${ }^{40}$ O. Zenaiev, ${ }^{40}$ R. Aggleton, ${ }^{41}$ S. Bein,${ }^{41}$ V. Blobel, ${ }^{41}$ M. Centis Vignali, ${ }^{41}$ T. Dreyer, ${ }^{41}$ E. Garutti, ${ }^{41}$ D. Gonzalez, ${ }^{41}$ J. Haller, ${ }^{41}$ A. Hinzmann, ${ }^{41}$ M. Hoffmann, ${ }^{41}$ A. Karavdina, ${ }^{41}$ G. Kasieczka, ${ }^{41}$ R. Klanner, ${ }^{41}$ R. Kogler, ${ }^{41}$ N. Kovalchuk, ${ }^{41}$ S. Kurz, ${ }^{41}$ D. Marconi, ${ }^{41}$ J. Multhaup, ${ }^{41}$ M. Niedziela, ${ }^{41}$ D. Nowatschin, ${ }^{41}$ T. Peiffer, ${ }^{41}$ A. Perieanu, ${ }^{41}$ A. Reimers, ${ }^{41}$ C. Scharf, ${ }^{41}$ P. Schleper, ${ }^{41}$ A. Schmidt, ${ }^{41}$ S. Schumann, ${ }^{41}$ J. Schwandt, ${ }^{41}$ J. Sonneveld, ${ }^{41}$ H. Stadie, ${ }^{41}$ G. Steinbrück, ${ }^{41}$ F. M. Stober ${ }^{41}$ M. Stöver, ${ }^{41}$ D. Troendle, ${ }^{41}$ E. Usai, ${ }^{41}$ A. Vanhoefer, ${ }^{41}$ B. Vormwald ${ }^{41}$ M. Akbiyik, ${ }^{42}$ C. Barth, ${ }^{42}$ M. Baselga, ${ }^{42}$ S. Baur, ${ }^{42}$ E. Butz,${ }^{42}$ R. Caspart, ${ }^{42}$ T. Chwalek,${ }^{42}$ F. Colombo, ${ }^{42}$ W. De Boer, ${ }^{42}$ A. Dierlamm, ${ }^{42}$ N. Faltermann, ${ }^{42}$ B. Freund, ${ }^{42}$ R. Friese,${ }^{42}$ M. Giffels,${ }^{42}$ M. A. Harrendorf, ${ }^{42}$ F. Hartmann, $, 2, p$ S. M. Heindl, ${ }^{42}$ U. Husemann, ${ }^{42}$ F. Kassel, ${ }^{42, p}$ S. Kudella, ${ }^{42}$ H. Mildner, ${ }^{42}$ M. U. Mozer, ${ }^{42}$ Th. Müller, ${ }^{42}$ M. Plagge, ${ }^{42}$ G. Quast, ${ }^{42}$ K. Rabbertz, ${ }^{42}$ M. Schröder, ${ }^{42}$ I. Shvetsov, ${ }^{42}$ G. Sieber, ${ }^{42}$ H. J. Simonis, ${ }^{42}$ R. Ulrich, ${ }^{42}$ S. Wayand,${ }^{42}$ M. Weber,${ }^{42}$ T. Weiler, ${ }^{42}$ S. Williamson, ${ }^{42}$ C. Wöhrmann, ${ }^{42}$ R. Wolf, ${ }^{42}$ G. Anagnostou, ${ }^{43}$ G. Daskalakis, ${ }^{43}$ T. Geralis, ${ }^{43}$ A. Kyriakis, ${ }^{43}$ D. Loukas, ${ }^{43}$ I. Topsis-Giotis, ${ }^{43}$ G. Karathanasis, ${ }^{44}$ S. Kesisoglou, ${ }^{44}$ A. Panagiotou, ${ }^{44}$ N. Saoulidou, ${ }^{44}$ E. Tziaferi, ${ }^{44}$ K. Kousouris,${ }^{45}$ I. Papakrivopoulos, ${ }^{45}$ I. Evangelou, ${ }^{46}$ C. Foudas, ${ }^{46}$ P. Gianneios, ${ }^{46}$ P. Katsoulis, ${ }^{46}$ P. Kokkas,${ }^{46}$ S. Mallios,${ }^{46}$ N. Manthos, ${ }^{46}$ I. Papadopoulos, ${ }^{46}$ E. Paradas,${ }^{46}$ J. Strologas, ${ }^{46}$ F. A. Triantis,${ }^{46}$ D. Tsitsonis, ${ }^{46}$ M. Csanad,${ }^{47}$ N. Filipovic, ${ }^{47}$ G. Pasztor, ${ }^{47}$ O. Surányi, ${ }^{47}$ G. I. Veres,${ }^{47, t}$ G. Bencze,${ }^{48}$ C. Hajdu, ${ }^{48}$ D. Horvath,${ }^{48, u}$ Á. Hunyadi, ${ }^{48}$ F. Sikler, ${ }^{48}$ V. Veszpremi ${ }^{48}$ G. Vesztergombi, ${ }^{48, t}$ T. Á. Vámi, ${ }^{48}$ N. Beni, ${ }^{49}$ S. Czellar, ${ }^{49}$ J. Karancsi, ${ }^{49, v}$ A. Makovec, ${ }^{49}$ J. Molnar, ${ }^{49}$ Z. Szillasi, ${ }^{49}$ M. Bartók, ${ }^{50, t}$ P. Raics,${ }^{50}$ Z. L. Trocsanyi, ${ }^{50}$ B. Ujvari, ${ }^{50}$ S. Choudhury, ${ }^{51}$ J. R. Komaragiri, ${ }^{51}$ S. Bahinipati, ${ }^{52, w}$ P. Mal,${ }^{52}$ K. Mandal, ${ }^{52}$ A. Nayak, ${ }^{52, x}$ D. K. Sahoo, ${ }^{52, w}$ N. Sahoo, ${ }^{52}$ S. K. Swain, ${ }^{52}$ S. Bansal, ${ }^{53}$ S. B. Beri, ${ }^{53}$ V. Bhatnagar, ${ }^{53}$ S. Chauhan, ${ }^{53}$ R. Chawla, ${ }^{53}$ N. Dhingra, ${ }^{53}$ R. Gupta, ${ }^{53}$ A. Kaur, ${ }^{53}$ M. Kaur, ${ }^{53}$ S. Kaur, ${ }^{53}$ R. Kumar, ${ }^{53}$ P. Kumari, ${ }^{53}$ A. Mehta, ${ }^{53}$ S. Sharma ${ }^{53}$ J. B. Singh,${ }^{53}$ G. Walia, ${ }^{53}$ Ashok Kumar, ${ }^{54}$ Aashaq Shah, ${ }^{54}$ A. Bhardwaj, ${ }^{54}$ B. C. Choudhary, ${ }^{54}$ R. B. Garg, ${ }^{54}$ S. Keshri, ${ }^{54}$ A. Kumar, ${ }^{54}$ S. Malhotra, ${ }^{54}$ M. Naimuddin,${ }^{54}$ K. Ranjan, ${ }^{54}$ R. Sharma, ${ }^{54}$ R. Bhardwaj, ${ }^{5, y}$ R. Bhattacharya, ${ }^{55}$ S. Bhattacharya ${ }^{55}$ U. Bhawandeep ${ }^{55, y}$ D. Bhowmik, ${ }^{55}$ S. Dey, ${ }^{55}$ S. Dutt, ${ }^{55, y}$ S. Dutta, ${ }^{55}$ S. Ghosh, ${ }^{55}$ N. Majumdar, ${ }^{55}$ K. Mondal,${ }^{55}$ S. Mukhopadhyay, ${ }^{55}$ S. Nandan,${ }^{55}$ A. Purohit, ${ }^{55}$ P. K. Rout, ${ }^{55}$ A. Roy, ${ }^{55}$ S. Roy Chowdhury, ${ }^{55}$ S. Sarkar, ${ }^{55}$ M. Sharan, ${ }^{55}$ B. Singh,${ }^{55}$ S. Thakur, ${ }^{55, y}$ P. K. Behera, ${ }^{56}$ R. Chudasama, ${ }^{57}$ D. Dutta, ${ }^{57}$ V. Jha, ${ }^{57}$ V. Kumar, ${ }^{57}$ A. K. Mohanty, ${ }^{57, p}$ P. K. Netrakanti, ${ }^{57}$ L. M. Pant,${ }^{57}$ P. Shukla, ${ }^{57}$ A. Topkar,${ }^{57}$ T. Aziz, ${ }^{58}$ S. Dugad,${ }^{58}$ B. Mahakud, ${ }^{58}$ S. Mitra, ${ }^{58}$ G. B. Mohanty, ${ }^{58}$ N. Sur, ${ }^{58}$ B. Sutar ${ }^{58}$ S. Banerjee ${ }^{59}$ S. Bhattacharya,${ }^{59}$ S. Chatterjee,${ }^{59}$ P. Das, ${ }^{59}$ M. Guchait,${ }^{59}$ Sa. Jain, ${ }^{59}$ S. Kumar, ${ }^{59}$ M. Maity, ${ }^{59, z}$ G. Majumder, ${ }^{59}$ K. Mazumdar, ${ }^{59}$ T. Sarkar, ${ }^{59, z}$ N. Wickramage, ${ }^{59, \text { aa }}$ S. Chauhan, ${ }^{60}$ S. Dube ${ }^{60}$ V. Hegde, ${ }^{60}$ A. Kapoor,${ }^{60}$ K. Kothekar,${ }^{60}$ S. Pandey,${ }^{60}$ A. Rane, ${ }^{60}$ S. Sharma ${ }^{60}$ S. Chenarani,${ }^{61, b b}$ E. Eskandari Tadavani, ${ }^{61}$ S. M. Etesami, ${ }^{61, b b}$ M. Khakzad, ${ }^{61}$ M. Mohammadi Najafabadi, ${ }^{61}$ M. Naseri, ${ }^{61}$ S. Paktinat Mehdiabadi, ${ }^{61, c c}$ F. Rezaei Hosseinabadi, ${ }^{61}$ B. Safarzadeh, ${ }^{61, d d}$ M. Zeinali, ${ }^{61}$ M. Felcini, ${ }^{62}$ M. Grunewald, ${ }^{62}$ M. Abbrescia ${ }^{63 a, 63 b}$ C. Calabria, ${ }^{63 a, 63 b}$ A. Colaleo, ${ }^{63 a}$ D. Creanza, ${ }^{63 a, 63 c}$ L. Cristella, ${ }^{63 a, 63 b}$ N. De Filippis, ${ }^{63 a, 63 c}$

M. De Palma, ${ }^{63 a, 63 b}$ A. Di Florio, ${ }^{63 a, 63 b}$ F. Errico, ${ }^{63 a, 63 b}$ L. Fiore, ${ }^{63 a}$ G. Iaselli, ${ }^{63 a, 63 c}$ S. Lezki, ${ }^{63 a, 63 b}$ G. Maggi, ${ }^{63,63}$

M. Maggi, ${ }^{63 a}$ B. Marangelli, ${ }^{63 a, 63 b}$ G. Miniello, ${ }^{63 a, 63 b}$ S. My, ${ }^{63 a, 63 b}$ S. Nuzzo, ${ }^{63 a, 63 b}$ A. Pompili, ${ }^{63 a, 63 b}$ G. Pugliese, ${ }^{63 a, 63 c}$ R. Radogna, ${ }^{63 a}$ A. Ranieri, ${ }^{63 a}$ G. Selvaggi, ${ }^{63 a, 63 b}$ A. Sharma ${ }^{63 a}$ L. Silvestris,${ }^{63 a, p}$ R. Venditti, ${ }^{63 a}$ P. Verwilligen,${ }^{63 a}$ G. Zito, ${ }^{63 a}$ G. Abbiendi, ${ }^{64 \mathrm{a}}$ C. Battilana, ${ }^{64,64 b}$ D. Bonacorsi, ${ }^{64 a, 64 b}$ L. Borgonovi, ${ }^{64 a, 64 b}$ S. Braibant-Giacomelli, ${ }^{64 a, 64 b}$

R. Campanini, ${ }^{64,64 b}$ P. Capiluppi, ${ }^{64 a, 64 b}$ A. Castro, ${ }^{64 a, 64 b}$ F. R. Cavallo, ${ }^{64 a}$ S. S. Chhibra, ${ }^{64 a, 64 b}$ G. Codispoti, ${ }^{64 a, 64 b}$ M. Cuffiani, ${ }^{64 a, 64 b}$ G. M. Dallavalle, ${ }^{64 a}$ F. Fabbri, ${ }^{64 a}$ A. Fanfani, ${ }^{64 a, 64 b}$ D. Fasanella, ${ }^{64 a, 64 b}$ P. Giacomelli, ${ }^{64 a}$ C. Grandi, ${ }^{64 a}$ L. Guiducci, ${ }^{64 a, 64 b}$ F. Iemmi, ${ }^{64 a}$ S. Marcellini, ${ }^{64 a}$ G. Masetti, ${ }^{64 a}$ A. Montanari, ${ }^{64 a}$ F. L. Navarria, ${ }^{64 a, 64 b}$ A. Perrotta, ${ }^{64 a}$ A. M. Rossi, ${ }^{64,64 b}$ T. Rovelli, ${ }^{64 a, 64 b}$ G. P. Siroli ${ }^{64 a, 64 b}$ N. Tosi,${ }^{64 a}$ S. Albergo, ${ }^{65 a, 65 b}$ S. Costa ${ }^{65 a, 65 b}$ A. Di Mattia, ${ }^{65 a}$ F. Giordano, ${ }^{65 a, 65 b}$ R. Potenza, ${ }^{65 a, 65 b}$ A. Tricomi, ${ }^{65 a, 65 b}$ C. Tuve,${ }^{65 a, 65 b}$ G. Barbagli, ${ }^{66 a}$ K. Chatterjee, ${ }^{66 a, 66 b}$ V. Ciulli, ${ }^{66 a, 66 b}$ C. Civinini, ${ }^{66 \mathrm{a}}$ R. D’Alessandro, ${ }^{66 a, 66 \mathrm{~b}}$ E. Focardi, ${ }^{66 \mathrm{a}, 66 \mathrm{~b}}$ G. Latino, ${ }^{66 \mathrm{a}}$ P. Lenzi, ${ }^{66,66 \mathrm{~b}}$ M. Meschini, ${ }^{66 \mathrm{a}}$ S. Paoletti, ${ }^{66 \mathrm{a}}$ L. Russo, ${ }^{66 a, e e}$ G. Sguazzoni, ${ }^{66 a}$ D. Strom, ${ }^{66 a}$ L. Viliani, ${ }^{66 a}$ L. Benussi ${ }^{67}$ S. Bianco,${ }^{67}$ F. Fabbri, ${ }^{67}$ D. Piccolo, ${ }^{67}$ F. Primavera, ${ }^{67, p}$ V. Calvelli, ${ }^{68 a, 68 b}$ F. Ferro, ${ }^{68 \mathrm{a}}$ F. Ravera, ${ }^{68 \mathrm{a}, 68 \mathrm{~b}}$ E. Robutti, ${ }^{68 \mathrm{a}}$ S. Tosi, ${ }^{68 \mathrm{a}, 68 \mathrm{~b}}$ A. Benaglia, ${ }^{69 \mathrm{a}}$ A. Beschi, ${ }^{69 \mathrm{~b}}$ L. Brianza, ${ }^{69 a, 69 b}$ F. Brivio, ${ }^{69 a, 69 b}$ V. Ciriolo,${ }^{69 a, 69 b, p}$ M. E. Dinardo,${ }^{69 a, 69 b}$ S. Fiorendi, ${ }^{69 a, 69 b}$ S. Gennai, ${ }^{69 a}$ A. Ghezzi, ${ }^{69 a, 69 b}$ P. Govoni ${ }^{69 a, 69 b}$ M. Malberti ${ }^{69 a, 69 b}$ S. Malvezzi ${ }^{69 a}$ R. A. Manzoni, ${ }^{69 a, 69 b}$ D. Menasce ${ }^{69 a}$ L. Moroni, ${ }^{69 a}$ M. Paganoni, ${ }^{69 a, 69 b}$ K. Pauwels, ${ }^{69 a, 69 b}$ D. Pedrini, ${ }^{69 a}$ S. Pigazzini, ${ }^{69 a, 69 b, f f}$ S. Ragazzi,${ }^{69 a, 69 b}$ T. Tabarelli de Fatis, ${ }^{69 a, 69 b}$ S. Buontempo, ${ }^{70 a}$ N. Cavallo, ${ }^{70 a, 70 c}$ S. Di Guida, ${ }^{70 a, 70 d, p}$ F. Fabozzi, ${ }^{70 a, 70 c}$ F. Fienga, ${ }^{70 a, 70 b}$ A. O. M. Iorio, ${ }^{70 a, 70 b}$ W. A. Khan, ${ }^{70 a}$ L. Lista, ${ }^{70 a}$ S. Meola, ${ }^{70 a, 70 d, p}$ P. Paolucci, ${ }^{70 a, p}$ C. Sciacca, ${ }^{70 a, 70 b}$ F. Thyssen, ${ }^{70 a}$ P. Azzi, ${ }^{71 a}$ N. Bacchetta, ${ }^{71 a}$ L. Benato, ${ }^{71 a, 71 b}$ D. Bisello, ${ }^{71 \mathrm{a}, 71 \mathrm{~b}}$ A. Boletti, ${ }^{71 \mathrm{a}, 71 \mathrm{~b}}$ R. Carlin, ${ }^{71 \mathrm{a}, 71 \mathrm{~b}}$ P. Checchia, ${ }^{71 \mathrm{a}}$ M. Dall'Osso, ${ }^{71 \mathrm{a}, 71 \mathrm{~b}}$ P. De Castro Manzano, ${ }^{71 \mathrm{a}}$ T. Dorigo, ${ }^{71 \mathrm{a}}$ 
F. Gasparini, ${ }^{71 \mathrm{a}, 71 \mathrm{~b}}$ U. Gasparini, ${ }^{71,71 \mathrm{~b}}$ A. Gozzelino, ${ }^{71 \mathrm{a}}$ S. Lacaprara, ${ }^{71 \mathrm{a}}$ P. Lujan, ${ }^{71 \mathrm{a}}$ M. Margoni, ${ }^{71 \mathrm{a}, 71 \mathrm{~b}}$

A. T. Meneguzzo, ${ }^{71 a, 71 b}$ M. Passaseo, ${ }^{71 a}$ N. Pozzobon, ${ }^{71 a, 71 b}$ P. Ronchese, ${ }^{71 a, 71 b}$ R. Rossin, ${ }^{71 a, 71 b}$ F. Simonetto, ${ }^{71 a, 71 b}$

A. Tiko, ${ }^{71 a}$ E. Torassa, ${ }^{71 a}$ S. Ventura, ${ }^{71 a}$ M. Zanetti, ${ }^{71 a, 71 b}$ P. Zotto, ${ }^{71 a, 71 b}$ A. Braghieri, ${ }^{72 a}$ A. Magnani, ${ }^{72 a}$ P. Montagna, ${ }^{72 a, 72 b}$

S. P. Ratti, ${ }^{72 a, 72 b}$ V. Re ${ }^{72 a}$ M. Ressegotti, ${ }^{72 a, 72 b}$ C. Riccardi, ${ }^{72 a, 72 b}$ P. Salvini, ${ }^{72 a}$ I. Vai, ${ }^{72 a, 72 b}$ P. Vitulo, ${ }^{72 a, 72 b}$

L. Alunni Solestizi, ${ }^{73 a, 73 b}$ M. Biasini, ${ }^{73 a, 73 b}$ G. M. Bilei, ${ }^{73 a}$ C. Cecchi, ${ }^{73 a, 73 b}$ D. Ciangottini, ${ }^{73 a, 73 b}$ L. Fanò, ${ }^{73 a, 73 b}$ P. Lariccia, ${ }^{73 a, 73 b}$ R. Leonardi, ${ }^{73 a, 73 b}$ E. Manoni,${ }^{73 a}$ G. Mantovani, ${ }^{73 a, 73 b}$ V. Mariani, ${ }^{73 a, 73 b}$ M. Menichelli, ${ }^{73 a}$ A. Rossi, ${ }^{73 a, 73 b}$ A. Santocchia, ${ }^{73 a, 73 b}$ D. Spiga ${ }^{73 a}$ K. Androsov, ${ }^{74 a}$ P. Azzurri, ${ }^{74 a, p}$ G. Bagliesi,${ }^{74 a}$ L. Bianchini,${ }^{74 a}$ T. Boccali,${ }^{74 a}$ L. Borrello,${ }^{74 a}$ R. Castaldi, ${ }^{74 a}$ M. A. Ciocci, ${ }^{74 a, 74 b}$ R. Dell'Orso, ${ }^{74 a}$ G. Fedi, ${ }^{74 a}$ L. Giannini, ${ }^{74 a, 74 c}$ A. Giassi, ${ }^{74 a}$ M. T. Grippo, ${ }^{74 a, e e}$ F. Ligabue, ${ }^{74 a, 74 c}$ T. Lomtadze, ${ }^{74 a}$ E. Manca, ${ }^{74 a, 74 c}$ G. Mandorlii, ${ }^{74 a, 74 c}$ A. Messineo, ${ }^{74 a, 74 b}$ F. Palla, ${ }^{74 a}$ A. Rizzi, ${ }^{74 a, 74 b}$ P. Spagnolo, ${ }^{74 a}$ R. Tenchini, ${ }^{74 a}$ G. Tonelli, ${ }^{74 a, 74 b}$ A. Venturi, ${ }^{74 a}$ P. G. Verdini, ${ }^{74 a}$ L. Barone, ${ }^{75 a, 75 b}$ F. Cavallari, ${ }^{75 a}$ M. Cipriani, ${ }^{75 a, 75 b}$ N. Daci, ${ }^{75 a}$ D. Del Re ${ }^{75 a, 75 b}$ E. Di Marco,${ }^{75 a, 75 b}$ M. Diemoz, ${ }^{75 a}$ S. Gelli, ${ }^{75 a, 75 b}$ E. Longo,${ }^{75 a, 75 b}$ F. Margaroli ${ }^{75 a, 75 b}$ B. Marzocchi, ${ }^{75 a, 75 b}$ P. Meridiani, ${ }^{75 a}$ G. Organtini ${ }^{75 a, 75 b}$ F. Pandolfi, ${ }^{75 a}$ R. Paramatti, ${ }^{75 a, 75 b}$ F. Preiato, ${ }^{75 a, 75 b}$ S. Rahatlou, ${ }^{75 a, 75 b}$ C. Rovelli, ${ }^{75 a}$ F. Santanastasio, ${ }^{75 a, 75 b}$ N. Amapane, ${ }^{76 a, 76 b}$ R. Arcidiacono, ${ }^{76 a, 76 c}$ S. Argiro, ${ }^{76 a, 76 b}$ M. Arneodo, ${ }^{76 a, 76 c}$ N. Bartosik, ${ }^{76 a}$ R. Bellan, ${ }^{76 a, 76 b}$ C. Biino,${ }^{76 a}$ N. Cartiglia, ${ }^{76 a}$ R. Castello, ${ }^{76 a, 76 b}$ F. Cenna, ${ }^{76 a, 76 b}$ M. Costa,${ }^{76 a, 76 b}$ R. Covarelli, ${ }^{76 a, 76 b}$ A. Degano, ${ }^{76 a, 76 b}$ N. Demaria, ${ }^{76 a}$ B. Kiani, ${ }^{76 a, 76 b}$ C. Mariotti, ${ }^{76 a}$ S. Maselli, ${ }^{76 a}$ E. Migliore, ${ }^{76 a, 76 b}$ V. Monaco, ${ }^{76 a, 76 b}$ E. Monteil, ${ }^{76 a, 76 b}$ M. Monteno, ${ }^{76 a}$ M. M. Obertino, ${ }^{76 a, 76 b}$ L. Pacher, ${ }^{76 a, 76 b}$ N. Pastrone, ${ }^{76 a}$ M. Pelliccioni, ${ }^{76 a}$ G. L. Pinna Angioni ${ }^{76 a, 76 b}$ A. Romero, ${ }^{76 a, 76 b}$ M. Ruspa ${ }^{76 a, 76 c}$ R. Sacchi, ${ }^{76 a, 76 b}$ K. Shchelina,${ }^{76 a, 76 b}$ V. Sola, ${ }^{76 a}$ A. Solano, ${ }^{76 a, 76 b}$ A. Staiano, ${ }^{76 a}$ P. Traczyk, ${ }^{76 a, 76 b}$ S. Belforte, ${ }^{77 a}$ M. Casarsa, ${ }^{77 a}$ F. Cossutti, ${ }^{77 a}$ G. Della Ricca, ${ }^{77 a, 77 b}$ A. Zanetti, ${ }^{77 a}$ D. H. Kim, ${ }^{78}$ G. N. Kim, ${ }^{78}$ M. S. Kim,${ }^{78}$ J. Lee, ${ }^{78}$ S. Lee, ${ }^{78}$ S. W. Lee, ${ }^{78}$ C. S. Moon, ${ }^{78}$ Y. D. Oh ${ }^{78}$ S. Sekmen, ${ }^{78}$ D. C. Son, ${ }^{78}$ Y. C. Yang,${ }^{78}$ H. Kim,${ }^{79}$ D. H. Moon, ${ }^{79}$ G. Oh,${ }^{79}$ J. A. Brochero Cifuentes,${ }^{80}$ J. Goh, ${ }^{80}$ T. J. Kim, ${ }^{80}$ S. Cho, ${ }^{81}$ S. Choi, ${ }^{81}$ Y. Go, ${ }^{81}$ D. Gyun, ${ }^{81}$ S. Ha, ${ }^{81}$ B. Hong, ${ }^{81}$ Y. Jo, ${ }^{81}$ Y. Kim, ${ }^{81}$ K. Lee, ${ }^{81}$ K. S. Lee,${ }^{81}$ S. Lee, ${ }^{81}$ J. Lim, ${ }^{81}$ S. K. Park, ${ }^{81}$ Y. Roh, ${ }^{81}$ J. Almond, ${ }^{82}$ J. Kim,${ }^{82}$ J. S. Kim, ${ }^{82}$ H. Lee, ${ }^{82}$ K. Lee, ${ }^{82}$ K. Nam, ${ }^{82}$ S. B. Oh, ${ }^{82}$ B. C. Radburn-Smith, ${ }^{82}$ S. h. Seo, ${ }^{82}$ U. K. Yang, ${ }^{82}$ H. D. Yoo, ${ }^{82}$ G. B. Yu, ${ }^{82}$ H. Kim, ${ }^{83}$ J. H. Kim, ${ }^{83}$ J. S. H. Lee, ${ }^{83}$ I. C. Park ${ }^{83}$ Y. Choi, ${ }^{84}$ C. Hwang, ${ }^{84}$ J. Lee, ${ }^{84}$ I. Yu, ${ }^{84}$ V. Dudenas, ${ }^{85}$ A. Juodagalvis,${ }^{85}$ J. Vaitkus, ${ }^{85}$ I. Ahmed, ${ }^{86}$ Z. A. Ibrahim, ${ }^{86}$ M. A. B. Md Ali, ${ }^{86, g g}$ F. Mohamad Idris, ${ }^{86, \text { hh }}$ W. A. T. Wan Abdullah, ${ }^{86}$ M. N. Yusli, ${ }^{86}$ Z. Zolkapli, ${ }^{86}$ R Reyes-Almanza, ${ }^{87}$ G. Ramirez-Sanchez, ${ }^{87}$ M. C. Duran-Osuna, ${ }^{87}$ H. Castilla-Valdez, ${ }^{87}$ E. De La Cruz-Burelo, ${ }^{87}$ I. Heredia-De La Cruz, ${ }^{87, i i}$ R. I. Rabadan-Trejo, ${ }^{87}$ R. Lopez-Fernandez, ${ }^{87}$ J. Mejia Guisao, ${ }^{87}$ A. Sanchez-Hernandez, ${ }^{87}$ S. Carrillo Moreno, ${ }^{88}$ C. Oropeza Barrera, ${ }^{88}$ F. Vazquez Valencia ${ }^{88}$ J. Eysermans,${ }^{89}$ I. Pedraza ${ }^{89}$ H. A. Salazar Ibarguen ${ }^{89}$ C. Uribe Estrada, ${ }^{89}$ A. Morelos Pineda, ${ }^{90}$ D. Krofcheck, ${ }^{91}$ P. H. Butler, ${ }^{92}$ A. Ahmad, ${ }^{93}$ M. Ahmad, ${ }^{93}$ Q. Hassan,${ }^{93}$ H. R. Hoorani, ${ }^{93}$ A. Saddique, ${ }^{93}$ M. A. Shah, ${ }^{93}$ M. Shoaib,${ }^{93}$ M. Waqas, ${ }^{93}$ H. Bialkowska, ${ }^{94}$ M. Bluj, ${ }^{94}$ B. Boimska, ${ }^{94}$ T. Frueboes,${ }^{94}$ M. Górski, ${ }^{94}$ M. Kazana, ${ }^{94}$ K. Nawrocki, ${ }^{94}$ M. Szleper,${ }^{94}$ P. Zalewski, ${ }^{94}$ K. Bunkowski, ${ }^{95}$ A. Byszuk, ${ }^{95, j j}$ K. Doroba, ${ }^{95}$ A. Kalinowski, ${ }^{95}$ M. Konecki, ${ }^{95}$ J. Krolikowski,${ }^{95}$ M. Misiura,${ }^{95}$ M. Olszewski,${ }^{95}$ A. Pyskir, ${ }^{95}$ M. Walczak,${ }^{95}$ P. Bargassa, ${ }^{96}$ C. Beirão Da Cruz E Silva, ${ }^{96}$ A. Di Francesco,${ }^{96}$ P. Faccioli, ${ }^{96}$ B. Galinhas,${ }^{96}$ M. Gallinaro, ${ }^{96}$ J. Hollar, ${ }^{96}$ N. Leonardo, ${ }^{96}$ L. Lloret Iglesias, ${ }^{96}$ M. V. Nemallapudi, ${ }^{96}$ J. Seixas, ${ }^{96}$ G. Strong,,${ }^{96}$ O. Toldaiev,${ }^{96}$ D. Vadruccio, ${ }^{96}$ J. Varela,${ }^{96}$ S. Afanasiev,${ }^{97}$ P. Bunin, ${ }^{97}$ M. Gavrilenko, ${ }^{97}$ I. Golutvin, ${ }^{97}$ I. Gorbunov, ${ }^{97}$ A. Kamenev, ${ }^{97}$ V. Karjavin, ${ }^{97}$ A. Lanev, ${ }^{97}$ A. Malakhov, ${ }^{97}$ V. Matveev, ${ }^{97, k k, 11}$ P. Moisenz, ${ }^{97}$ V. Palichik, ${ }^{97}$ V. Perelygin, ${ }^{97}$ S. Shmatov, ${ }^{97}$ S. Shulha, ${ }^{97}$ N. Skatchkov, ${ }^{97}$ V. Smirnov, ${ }^{97}$ N. Voytishin, ${ }^{97}$ A. Zarubin, ${ }^{97}$ Y. Ivanov, ${ }^{98}$ V. Kim,,${ }^{98, m m}$ E. Kuznetsova, ${ }^{98, n n}$ P. Levchenko, ${ }^{98}$ V. Murzin, ${ }^{98}$ V. Oreshkin, ${ }^{98}$ I. Smirnov, ${ }^{98}$ D. Sosnov, ${ }^{98}$ V. Sulimov, ${ }^{98}$ L. Uvarov, ${ }^{98}$ S. Vavilov, ${ }^{98}$ A. Vorobyev, ${ }^{98}$ Yu. Andreev, ${ }^{99}$ A. Dermenev, ${ }^{99}$ S. Gninenko, ${ }^{99}$ N. Golubev, ${ }^{99}$ A. Karneyeu, ${ }^{99}$ M. Kirsanov, ${ }^{99}$ N. Krasnikov, ${ }^{99}$ A. Pashenkov, ${ }^{99}$ D. Tlisov, ${ }^{99}$ A. Toropin,${ }^{99}$ V. Epshteyn, ${ }^{100}$ V. Gavrilov, ${ }^{100}$ N. Lychkovskaya, ${ }^{100}$ V. Popov, ${ }^{100}$ I. Pozdnyakov, ${ }^{100}$ G. Safronov, ${ }^{100}$ A. Spiridonov, ${ }^{100}$ A. Stepennov, ${ }^{100}$ V. Stolin, ${ }^{100}$ M. Toms ${ }^{100}$ E. Vlasov, ${ }^{100}$ A. Zhokin, ${ }^{100}$ T. Aushev, ${ }^{101}$ A. Bylinkin, ${ }^{101,11}$ M. Chadeeva, ${ }^{102,00}$ P. Parygin,,${ }^{102}$ D. Philippov, ${ }^{102}$ S. Polikarpov, ${ }^{102}$ E. Popova,${ }^{102}$ V. Rusinov, ${ }^{102}$ V. Andreev ${ }^{103}$ M. Azarkin, ${ }^{103,11}$ I. Dremin,,${ }^{103,11}$

M. Kirakosyan, ${ }^{103,11}$ S. V. Rusakov, ${ }^{103}$ A. Terkulov, ${ }^{103}$ A. Baskakov, ${ }^{104}$ A. Belyaev, ${ }^{104}$ E. Boos, ${ }^{104}$ V. Bunichev, ${ }^{104}$ M. Dubinin, ${ }^{104, p p}$ L. Dudko, ${ }^{104}$ A. Ershov, ${ }^{104}$ A. Gribushin, ${ }^{104}$ V. Klyukhin, ${ }^{104}$ O. Kodolova, ${ }^{104}$ I. Lokhtin, ${ }^{104}$ I. Miagkov, ${ }^{104}$ S. Obraztsov, ${ }^{104}$ S. Petrushanko, ${ }^{104}$ V. Savrin,${ }^{104}$ V. Blinov, ${ }^{105, q q}$ D. Shtol, ${ }^{105, q q}$ Y. Skovpen, ${ }^{105, q q}$ I. Azhgirey, ${ }^{106}$ I. Bayshev, ${ }^{106}$ S. Bitioukov, ${ }^{106}$ D. Elumakhov, ${ }^{106}$ A. Godizov, ${ }^{106}$ V. Kachanov, ${ }^{106}$ A. Kalinin, ${ }^{106}$ D. Konstantinov, ${ }^{106}$ P. Mandrik,${ }^{106}$ V. Petrov,${ }^{106}$ R. Ryutin, ${ }^{106}$ A. Sobol, ${ }^{106}$ S. Troshin, ${ }^{106}$ N. Tyurin, ${ }^{106}$ A. Uzunian, ${ }^{106}$ A. Volkov, ${ }^{106}$ A. Babaev ${ }^{107}$ P. Adzic, ${ }^{108, r r}$ P. Cirkovic, ${ }^{108}$ D. Devetak, ${ }^{108}$ M. Dordevic, ${ }^{108}$ J. Milosevic, ${ }^{108}$ J. Alcaraz Maestre, ${ }^{109}$ I. Bachiller, ${ }^{109}$ M. Barrio Luna, ${ }^{109}$ M. Cerrada, ${ }^{109}$ N. Colino, ${ }^{109}$ B. De La Cruz,${ }^{109}$ A. Delgado Peris, ${ }^{109}$ 
C. Fernandez Bedoya, ${ }^{109}$ J. P. Fernández Ramos, ${ }^{109}$ J. Flix, ${ }^{109}$ M. C. Fouz ${ }^{109}$ O. Gonzalez Lopez, ${ }^{109}$ S. Goy Lopez, ${ }^{109}$ J. M. Hernandez, ${ }^{109}$ M. I. Josa, ${ }^{109}$ D. Moran, ${ }^{109}$ A. Pérez-Calero Yzquierdo, ${ }^{109}$ J. Puerta Pelayo, ${ }^{109}$ I. Redondo, ${ }^{109}$

L. Romero, ${ }^{109}$ M. S. Soares, ${ }^{109}$ A. Triossi, ${ }^{109}$ A. Álvarez Fernández, ${ }^{109}$ C. Albajar, ${ }^{110}$ J. F. de Trocóniz, ${ }^{110}$ J. Cuevas, ${ }^{111}$ C. Erice, ${ }^{111}$ J. Fernandez Menendez, ${ }^{111}$ S. Folgueras, ${ }^{111}$ I. Gonzalez Caballero, ${ }^{111}$ J. R. González Fernández,${ }^{11}$ E. Palencia Cortezon, ${ }^{111}$ S. Sanchez Cruz, ${ }^{111}$ P. Vischia, ${ }^{111}$ J. M. Vizan Garcia, ${ }^{111}$ I. J. Cabrillo, ${ }^{112}$ A. Calderon, ${ }^{112}$ B. Chazin Quero, ${ }^{112}$ J. Duarte Campderros, ${ }^{112}$ M. Fernandez, ${ }^{112}$ P. J. Fernández Manteca, ${ }^{112}$ J. Garcia-Ferrero, ${ }^{112}$ A. García Alonso, ${ }^{112}$ G. Gomez, ${ }^{112}$ A. Lopez Virto, ${ }^{112}$ J. Marco, ${ }^{112}$ C. Martinez Rivero, ${ }^{112}$ P. Martinez Ruiz del Arbol, ${ }^{112}$ F. Matorras, ${ }^{112}$ J. Piedra Gomez,${ }^{112}$ C. Prieels, ${ }^{112}$ T. Rodrigo ${ }^{112}$ A. Ruiz-Jimeno, ${ }^{112}$ L. Scodellaro, ${ }^{112}$ N. Trevisani, ${ }^{112}$ I. Vila, ${ }^{112}$ R. Vilar Cortabitarte, ${ }^{112}$ D. Abbaneo, ${ }^{113}$ B. Akgun, ${ }^{113}$ E. Auffray, ${ }^{113}$ P. Baillon, ${ }^{113}$ A. H. Ball, ${ }^{113}$ D. Barney, ${ }^{113}$ J. Bendavid, ${ }^{113}$ M. Bianco, ${ }^{113}$ A. Bocci, ${ }^{113}$ C. Botta, ${ }^{113}$ T. Camporesi,${ }^{113}$ M. Cepeda, ${ }^{113}$ G. Cerminara, ${ }^{113}$ E. Chapon, ${ }^{113}$ Y. Chen, ${ }^{113}$ D. d'Enterria, ${ }^{113}$ A. Dabrowski, ${ }^{113}$ V. Daponte, ${ }^{113}$ A. David, ${ }^{113}$ M. De Gruttola ${ }^{113}$ A. De Roeck, ${ }^{113}$ N. Deelen, ${ }^{113}$ M. Dobson, ${ }^{113}$ T. du Pree, ${ }^{113}$ M. Dünser,${ }^{113}$ N. Dupont, ${ }^{113}$ A. Elliott-Peisert, ${ }^{113}$ P. Everaerts, ${ }^{113}$ F. Fallavollita, ${ }^{113, s s}$ G. Franzoni, ${ }^{113}$ J. Fulcher ${ }^{113}$ W. Funk, ${ }^{113}$ D. Gigi,${ }^{113}$ A. Gilbert, ${ }^{113}$ K. Gill,${ }^{113}$ F. Glege,${ }^{113}$ D. Gulhan, ${ }^{113}$ J. Hegeman, ${ }^{113}$ V. Innocente, ${ }^{113}$ A. Jafari, ${ }^{113}$ P. Janot, ${ }^{113}$ O. Karacheban, ${ }^{11,, \mathrm{~S}}$ J. Kieseler, ${ }^{113}$ V. Knünz, ${ }^{113}$ A. Kornmayer, ${ }^{113}$ M. Krammer, ${ }^{13, b}$ C. Lange, ${ }^{113}$ P. Lecoq, ${ }^{113}$ C. Lourenço, ${ }^{113}$ M. T. Lucchini, ${ }^{113}$ L. Malgeri, ${ }^{113}$ M. Mannelli, ${ }^{113}$ A. Martelli, ${ }^{113}$ F. Meijers, ${ }^{113}$ J. A. Merlin, ${ }^{113}$ S. Mersi, ${ }^{113}$ E. Meschi, ${ }^{113}$ P. Milenovic, ${ }^{113, t t}$ F. Moortgat, ${ }^{113}$ M. Mulders, ${ }^{113}$ H. Neugebauer, ${ }^{113}$ J. Ngadiuba, ${ }^{113}$ S. Orfanelli, ${ }^{113}$ L. Orsini, ${ }^{113}$ F. Pantaleo, ${ }^{11, p}$ L. Pape, ${ }^{113}$ E. Perez, ${ }^{113}$ M. Peruzzi, ${ }^{113}$ A. Petrilli, ${ }^{113}$ G. Petrucciani, ${ }^{113}$ A. Pfeiffer ${ }^{113}$ M. Pierini, ${ }^{113}$ F. M. Pitters, ${ }^{113}$ D. Rabady, ${ }^{113}$ A. Racz, ${ }^{113}$ T. Reis, ${ }^{113}$ G. Rolandi, ${ }^{113 \text {, uu }}$ M. Rovere ${ }^{113}$ H. Sakulin, ${ }^{113}$ C. Schäfer,${ }^{113}$ C. Schwick,,${ }^{113}$ M. Seidel,${ }^{113}$ M. Selvaggi, ${ }^{113}$ A. Sharma, ${ }^{113}$ P. Silva, ${ }^{113}$ P. Sphicas, ${ }^{113, \mathrm{vv}}$ A. Stakia, ${ }^{113}$ J. Steggemann, ${ }^{113}$ M. Stoye, ${ }^{113}$ M. Tosi, ${ }^{113}$ D. Treille, ${ }^{113}$ A. Tsirou, ${ }^{113}$ V. Veckalns, ${ }^{113, \text { ww }}$ M. Verweij, ${ }^{113}$ W. D. Zeuner, ${ }^{113}$ W. Bertl, ${ }^{114, a}$ L. Caminada, ${ }^{114, x x}$ K. Deiters, ${ }^{114}$ W. Erdmann, ${ }^{114}$ R. Horisberger, ${ }^{114}$ Q. Ingram, ${ }^{114}$ H. C. Kaestli, ${ }^{114}$ D. Kotlinski, ${ }^{114}$ U. Langenegger,${ }^{114}$ T. Rohe, ${ }^{114}$ S. A. Wiederkehr, ${ }^{114}$ M. Backhaus, ${ }^{115}$ L. Bäni, ${ }^{115}$ P. Berger, ${ }^{115}$ B. Casal, ${ }^{115}$ G. Dissertori, ${ }^{115}$ M. Dittmar, ${ }^{115}$ M. Donegà,${ }^{115}$ C. Dorfer, ${ }^{115}$ C. Grab, ${ }^{115}$ C. Heidegger, ${ }^{115}$ D. Hits, ${ }^{115}$ J. Hoss,${ }^{115}$ T. Klijnsma, ${ }^{115}$ W. Lustermann, ${ }^{115}$ M. Marionneau, ${ }^{115}$ M. T. Meinhard, ${ }^{115}$ D. Meister, ${ }^{115}$ F. Micheli, ${ }^{115}$ P. Musella, ${ }^{115}$ F. Nessi-Tedaldi, ${ }^{115}$ J. Pata, ${ }^{115}$ F. Pauss, ${ }^{115}$ G. Perrin, ${ }^{115}$ L. Perrozzi, ${ }^{115}$ M. Quittnat, ${ }^{115}$ M. Reichmann, ${ }^{115}$ D. A. Sanz Becerra, ${ }^{115}$ M. Schönenberger, ${ }^{15}$ L. Shchutska, ${ }^{115}$ V. R. Tavolaro, ${ }^{115}$

K. Theofilatos, ${ }^{115}$ M. L. Vesterbacka Olsson, ${ }^{115}$ R. Wallny, ${ }^{115}$ D. H. Zhu, ${ }^{115}$ T. K. Aarrestad, ${ }^{116}$ C. Amsler, ${ }^{116, y y}$ D. Brzhechko, ${ }^{116}$ M. F. Canelli, ${ }^{116}$ A. De Cosa,${ }^{116}$ R. Del Burgo, ${ }^{116}$ S. Donato, ${ }^{116}$ C. Galloni, ${ }^{116}$ T. Hreus, ${ }^{116}$ B. Kilminster, ${ }^{116}$ I. Neutelings, ${ }_{116}^{16}$ D. Pinna, ${ }^{116}$ G. Rauco, ${ }^{116}$ P. Robmann, ${ }^{116}$ D. Salerno, ${ }^{116}$ K. Schweiger, ${ }^{116}$ C. Seitz, ${ }^{116}$ Y. Takahashi, ${ }^{116}$ A. Zucchetta, ${ }^{116}$ V. Candelise, ${ }^{117}$ Y. H. Chang, ${ }^{117}$ K. y. Cheng, ${ }^{117}$ T. H. Doan, ${ }^{117}$ Sh. Jain, ${ }^{117}$ R. Khurana, ${ }^{117}$ C. M. Kuo, ${ }^{117}$ W. Lin, ${ }^{117}$ A. Pozdnyakov, ${ }^{117}$ S. S. Yu, ${ }^{117}$ Arun Kumar, ${ }^{118}$ P. Chang, ${ }^{118}$ Y. Chao, ${ }^{118}$ K. F. Chen, ${ }^{118}$ P. H. Chen, ${ }^{118}$ F. Fiori, ${ }^{118}$ W.-S. Hou, ${ }^{118}$ Y. Hsiung, ${ }^{118}$ Y. F. Liu, ${ }^{118}$ R.-S. Lu, ${ }^{118}$ E. Paganis, ${ }^{118}$ A. Psallidas, ${ }^{118}$ A. Steen, ${ }^{118}$ J. f. Tsai, ${ }^{118}$ B. Asavapibhop, ${ }^{119}$ K. Kovitanggoon, ${ }^{119}$ G. Singh,${ }^{119}$ N. Srimanobhas, ${ }^{119}$ A. Bat, ${ }^{120}$ F. Boran, ${ }^{120}$ S. Cerci, ${ }^{120, z z}$ S. Damarseckin, ${ }^{120}$ Z. S. Demiroglu, ${ }^{120}$ C. Dozen, ${ }^{120}$ I. Dumanoglu, ${ }^{120}$ S. Girgis, ${ }^{120}$ G. Gokbulut ${ }^{120}$ Y. Guler,${ }^{120}$ I. Hos, ${ }^{120 \text {,aaa }}$ E. E. Kangal, ${ }^{120, b b b}$ O. Kara, ${ }^{120}$ A. Kayis Topaksu, ${ }^{120}$ U. Kiminsu, ${ }^{120}$ M. Oglakci, ${ }^{120}$ G. Onengut, ${ }^{120}$ K. Ozdemir, ${ }^{120, c c c}$ D. Sunar Cerci, ${ }^{120, z z}$ B. Tali, ${ }^{120, z z}$ U. G. Tok, ${ }^{120}$ S. Turkcapar, ${ }^{120}$ I. S. Zorbakir, ${ }^{120}$ C. Zorbilmez, ${ }^{120}$ G. Karapinar, ${ }^{121, \text { ddd }}$ K. Ocalan, ${ }^{121, \text { eee }}$ M. Yalvac, ${ }^{121}$ M. Zeyrek, ${ }^{121}$ E. Gülmez, ${ }^{122}$ M. Kaya, ${ }^{122, f f}$ O. Kaya, ${ }^{122, g g g}$ S. Tekten, ${ }^{122}$ E. A. Yetkin, ${ }^{122, \text { hhh }}$ M. N. Agaras, ${ }^{123}$ S. Atay, ${ }^{123}$ A. Cakir, ${ }^{123}$ K. Cankocak, ${ }^{123, i i i}$ Y. Komurcu, ${ }^{123}$ B. Grynyov, ${ }^{124}$ L. Levchuk, ${ }^{125}$ F. Ball, ${ }^{126}$ L. Beck, ${ }^{126}$ J. J. Brooke, ${ }^{126}$ D. Burns,${ }^{126}$ E. Clement, ${ }^{126}$ D. Cussans, ${ }^{126}$ O. Davignon, ${ }^{126}$ H. Flacher, ${ }^{126}$ J. Goldstein, ${ }^{126}$ G. P. Heath, ${ }^{126}$ H. F. Heath, ${ }^{126}$ L. Kreczko, ${ }^{126}$ D. M. Newbold, ${ }^{126, j j \mathrm{j}}$ S. Paramesvaran, ${ }^{126}$ T. Sakuma, ${ }^{126}$

S. Seif El Nasr-storey, ${ }^{126}$ D. Smith, ${ }^{126}$ V. J. Smith, ${ }^{126}$ K. W. Bell, ${ }^{127}$ A. Belyaev, ${ }^{127, k k k}$ C. Brew, ${ }^{127}$ R. M. Brown, ${ }^{127}$ L. Calligaris, ${ }^{127}$ D. Cieri, ${ }^{127}$ D. J. A. Cockerill, ${ }^{127}$ J. A. Coughlan, ${ }^{127}$ K. Harder, ${ }^{127}$ S. Harper, ${ }^{127}$ J. Linacre,${ }^{127}$ E. Olaiya, ${ }^{127}$ D. Petyt, ${ }^{127}$ C. H. Shepherd-Themistocleous, ${ }^{127}$ A. Thea ${ }^{127}$ I. R. Tomalin, ${ }^{127}$ T. Williams, ${ }^{127}$ W. J. Womersley, ${ }^{127}$ G. Auzinger, ${ }^{128}$ R. Bainbridge, ${ }^{128}$ P. Bloch, ${ }^{128}$ J. Borg, ${ }^{128}$ S. Breeze, ${ }^{128}$ O. Buchmuller, ${ }^{128}$ A. Bundock, ${ }^{128}$ S. Casasso, ${ }^{128}$ D. Colling, ${ }^{128}$ L. Corpe, ${ }^{128}$ P. Dauncey, ${ }^{128}$ G. Davies, ${ }^{128}$ M. Della Negra, ${ }^{128}$ R. Di Maria, ${ }^{128}$ A. Elwood, ${ }^{128}$ Y. Haddad, ${ }^{128}$ G. Hall, ${ }^{128}$ G. Iles, ${ }^{128}$ T. James, ${ }^{128}$ M. Komm, ${ }^{128}$ R. Lane, ${ }^{128}$ C. Laner, ${ }^{128}$ L. Lyons, ${ }^{128}$ A.-M. Magnan, ${ }^{128}$ S. Malik, ${ }^{128}$ L. Mastrolorenzo, ${ }^{128}$ T. Matsushita, ${ }^{128}$ J. Nash, ${ }^{128,111}$ A. Nikitenko, ${ }^{128, \mathrm{~h}}$ V. Palladino, ${ }^{128}$ M. Pesaresi, ${ }^{128}$ A. Richards, ${ }^{128}$ A. Rose, ${ }^{128}$ E. Scott, ${ }^{128}$ C. Seez, ${ }^{128}$ A. Shtipliyski, ${ }^{128}$ T. Strebler, ${ }^{128}$ S. Summers, ${ }^{128}$ A. Tapper,${ }^{128}$ K. Uchida, ${ }^{128}$ M. Vazquez Acosta, ${ }^{128, \mathrm{mmm}}$ T. Virdee, ${ }^{128, \mathrm{p}}$ N. Wardle, ${ }^{128}$ D. Winterbottom, ${ }^{128}$ J. Wright, ${ }^{128}$ S. C. Zenz, ${ }^{128}$ J. E. Cole, ${ }^{129}$ 
P. R. Hobson, ${ }^{129}$ A. Khan, ${ }^{129}$ P. Kyberd, ${ }^{129}$ A. Morton, ${ }^{129}$ I. D. Reid, ${ }^{129}$ L. Teodorescu, ${ }^{129}$ S. Zahid, ${ }^{129}$ A. Borzou, ${ }^{130}$ K. Call, ${ }^{130}$ J. Dittmann, ${ }^{130}$ K. Hatakeyama, ${ }^{130}$ H. Liu, ${ }^{130}$ N. Pastika, ${ }^{130}$ C. Smith,${ }^{130}$ R. Bartek, ${ }^{131}$ A. Dominguez, ${ }^{131}$ A. Buccilli, ${ }^{132}$ S. I. Cooper, ${ }^{132}$ C. Henderson, ${ }^{132}$ P. Rumerio, ${ }^{132}$ C. West, ${ }^{132}$ D. Arcaro, ${ }^{133}$ A. Avetisyan, ${ }^{133}$ T. Bose, ${ }^{133}$ D. Gastler, ${ }^{133}$ D. Rankin, ${ }^{133}$ C. Richardson, ${ }^{133}$ J. Rohlf, ${ }^{133}$ L. Sulak, ${ }^{133}$ D. Zou, ${ }^{133}$ G. Benelli, ${ }^{134}$ D. Cutts, ${ }^{134}$ M. Hadley, ${ }^{134}$ J. Hakala, ${ }^{134}$ U. Heintz, ${ }^{134}$ J. M. Hogan, ${ }^{134, n n n}$ K. H. M. Kwok, ${ }^{134}$ E. Laird ${ }^{134}$ G. Landsberg, ${ }^{134}$ J. Lee, ${ }^{134}$ Z. Mao, ${ }^{134}$ M. Narain, ${ }^{134}$ J. Pazzini, ${ }^{134}$ S. Piperov ${ }^{134}$ S. Sagir, ${ }^{134}$ R. Syarif ${ }^{134}$ D. Yu,${ }^{134}$ R. Band, ${ }^{135}$ C. Brainerd, ${ }^{135}$ R. Breedon, ${ }^{135}$ D. Burns, ${ }^{135}$ M. Calderon De La Barca Sanchez, ${ }^{135}$ M. Chertok, ${ }^{135}$ J. Conway, ${ }^{135}$ R. Conway, ${ }^{135}$ P. T. Cox, ${ }^{135}$ R. Erbacher, ${ }^{135}$ C. Flores, ${ }^{135}$ G. Funk, ${ }^{135}$ W. Ko, ${ }^{135}$ R. Lander, ${ }^{135}$ C. Mclean, ${ }^{135}$ M. Mulhearn, ${ }^{135}$ D. Pellett, ${ }^{135}$ J. Pilot,${ }^{135}$ S. Shalhout, ${ }^{135}$ M. Shi, ${ }^{135}$ J. Smith, ${ }^{135}$ D. Stolp, ${ }^{135}$ D. Taylor, ${ }^{135}$ K. Tos, ${ }^{135}$ M. Tripathi, ${ }^{135}$ Z. Wang, ${ }^{135}$ F. Zhang, ${ }^{135}$ M. Bachtis, ${ }^{136}$ C. Bravo, ${ }^{136}$ R. Cousins, ${ }^{136}$ A. Dasgupta, ${ }^{136}$ A. Florent, ${ }^{136}$ J. Hauser ${ }^{136}$ M. Ignatenko, ${ }^{136}$ N. Mccoll, ${ }^{136}$ S. Regnard, ${ }^{136}$ D. Saltzberg, ${ }^{136}$ C. Schnaible, ${ }^{136}$ V. Valuev, ${ }^{136}$ E. Bouvier, ${ }^{137}$ K. Burt, ${ }^{137}$ R. Clare, ${ }^{137}$ J. Ellison, ${ }^{137}$ J. W. Gary, ${ }^{137}$ S. M. A. Ghiasi Shirazi, ${ }^{137}$ G. Hanson, ${ }^{137}$ G. Karapostoli, ${ }^{137}$ E. Kennedy, ${ }^{137}$ F. Lacroix, ${ }^{137}$ O. R. Long, ${ }^{137}$ M. Olmedo Negrete, ${ }^{137}$ M. I. Paneva, ${ }^{137}$ W. Si,${ }^{137}$ L. Wang, ${ }^{137}$ H. Wei, ${ }^{137}$ S. Wimpenny, ${ }^{137}$ B. R. Yates, ${ }^{137}$ J. G. Branson, ${ }^{138}$ S. Cittolin, ${ }^{138}$ M. Derdzinski, ${ }^{138}$ R. Gerosa, ${ }^{138}$ D. Gilbert, ${ }^{138}$ B. Hashemi, ${ }^{138}$ A. Holzner, ${ }^{138}$ D. Klein, ${ }^{138}$ G. Kole, ${ }^{138}$ V. Krutelyov, ${ }^{138}$ J. Letts, ${ }^{138}$ M. Masciovecchio, ${ }^{138}$ D. Olivito, ${ }^{138}$ S. Padhi, ${ }^{138}$ M. Pieri, ${ }^{138}$ M. Sani, ${ }^{138}$ V. Sharma, ${ }^{138}$ S. Simon, ${ }^{138}$ M. Tadel, ${ }^{138}$ A. Vartak, ${ }^{138}$ S. Wasserbaech, ${ }^{138,000}$ J. Wood, ${ }^{138}$ F. Würthwein, ${ }^{138}$ A. Yagil, ${ }^{138}$

G. Zevi Della Porta, ${ }^{138}$ N. Amin,${ }^{139}$ R. Bhandari, ${ }^{139}$ J. Bradmiller-Feld, ${ }^{139}$ C. Campagnari, ${ }^{139}$ M. Citron, ${ }^{139}$ A. Dishaw, ${ }^{139}$ V. Dutta, ${ }^{139}$ M. Franco Sevilla, ${ }^{139}$ L. Gouskos, ${ }^{139}$ R. Heller, ${ }^{139}$ J. Incandela, ${ }^{139}$ A. Ovcharova, ${ }^{139}$ H. Qu, ${ }^{139}$ J. Richman, ${ }^{139}$ D. Stuart, ${ }^{139}$ I. Suarez, ${ }^{139}$ J. Yoo, ${ }^{139}$ D. Anderson, ${ }^{140}$ A. Bornheim, ${ }^{140}$ J. Bunn, ${ }^{140}$ J. M. Lawhorn, ${ }^{140}$ H. B. Newman, ${ }^{140}$ T. Q. Nguyen, ${ }^{140}$ C. Pena, ${ }^{140}$ M. Spiropulu, ${ }^{140}$ J. R. Vlimant, ${ }^{140}$ R. Wilkinson, ${ }^{140}$ S. Xie, ${ }^{140}$ Z. Zhang, ${ }^{140}$ R. Y. Zhu, ${ }^{140}$ M. B. Andrews, ${ }^{141}$ T. Ferguson, ${ }^{141}$ T. Mudholkar, ${ }^{141}$ M. Paulini, ${ }^{141}$ J. Russ, ${ }^{141}$ M. Sun, ${ }^{141}$ H. Vogel,${ }^{141}$ I. Vorobiev, ${ }^{141}$ M. Weinberg, ${ }^{141}$ J. P. Cumalat, ${ }^{142}$ W. T. Ford, ${ }^{142}$ F. Jensen, ${ }^{142}$ A. Johnson, ${ }^{142}$ M. Krohn, ${ }^{142}$ S. Leontsinis, ${ }^{142}$ E. Macdonald, ${ }^{142}$ T. Mulholland, ${ }^{142}$ K. Stenson, ${ }^{142}$ K. A. Ulmer, ${ }^{142}$ S. R. Wagner, ${ }^{142}$ J. Alexander, ${ }^{143}$ J. Chaves,${ }^{143}$ Y. Cheng, ${ }^{143}$ J. Chu, ${ }^{143}$ A. Datta, ${ }^{143}$ K. Mcdermott, ${ }^{143}$ N. Mirman, ${ }^{143}$ J. R. Patterson, ${ }^{143}$ D. Quach, ${ }^{143}$ A. Rinkevicius, ${ }^{143}$ A. Ryd, ${ }^{143}$ L. Skinnari, ${ }^{143}$ L. Soffi, ${ }^{143}$ S. M. Tan, ${ }^{143}$ Z. Tao, ${ }^{143}$ J. Thom, ${ }^{143}$ J. Tucker, ${ }^{143}$ P. Wittich, ${ }^{143}$ M. Zientek, ${ }^{143}$ S. Abdullin, ${ }^{144}$ M. Albrow, ${ }^{144}$ M. Alyari, ${ }^{144}$ G. Apollinari, ${ }^{144}$ A. Apresyan, ${ }^{144}$ A. Apyan, ${ }^{144}$ S. Banerjee, ${ }^{144}$ L. A. T. Bauerdick, ${ }^{144}$ A. Beretvas, ${ }^{144}$ J. Berryhill, ${ }^{144}$ P. C. Bhat, ${ }^{144}$ G. Bolla, ${ }^{144, a}$ K. Burkett,,${ }^{144}$ J. N. Butler, ${ }^{144}$ A. Canepa,${ }^{144}$ G. B. Cerati, ${ }^{144}$ H. W. K. Cheung, ${ }^{144}$ F. Chlebana, ${ }^{144}$ M. Cremonesi ${ }^{144}$ J. Duarte, ${ }^{144}$ V. D. Elvira, ${ }^{144}$ J. Freeman, ${ }^{144}$ Z. Gecse, ${ }^{144}$ E. Gottschalk, ${ }^{144}$ L. Gray, ${ }^{144}$ D. Green,${ }^{144}$ S. Grünendahl, ${ }^{144}$ O. Gutsche, ${ }^{144}$ J. Hanlon, ${ }^{144}$ R. M. Harris ${ }^{144}$ S. Hasegawa, ${ }^{144}$ J. Hirschauer, ${ }^{144}$ Z. Hu, ${ }^{144}$ B. Jayatilaka, ${ }^{144}$ S. Jindariani, ${ }^{144}$ M. Johnson, ${ }^{144}$ U. Joshi, ${ }^{144}$ B. Klima, ${ }^{144}$ M. J. Kortelainen, ${ }^{144}$ B. Kreis ${ }^{144}$ S. Lammel, ${ }^{144}$ D. Lincoln, ${ }^{144}$ R. Lipton, ${ }^{144}$ M. Liu, ${ }^{144}$ T. Liu, ${ }^{144}$ R. Lopes De Sá, ${ }^{144}$ J. Lykken, ${ }^{144}$ K. Maeshima, ${ }^{144}$ N. Magini, ${ }^{144}$ J. M. Marraffino, ${ }^{144}$ D. Mason, ${ }^{144}$ P. McBride, ${ }^{144}$ P. Merkel,${ }^{144}$ S. Mrenna,${ }^{144}$ S. Nahn, ${ }^{144}$ V. O’Dell, ${ }^{144}$ K. Pedro, ${ }^{144}$ O. Prokofyev, ${ }^{144}$ G. Rakness, ${ }^{144}$ L. Ristori, ${ }^{144}$ A. Savoy-Navarro, ${ }^{144, p p p}$ B. Schneider, ${ }^{144}$ E. Sexton-Kennedy, ${ }^{144}$ A. Soha, ${ }^{144}$ W. J. Spalding, ${ }^{144}$ L. Spiegel, ${ }^{144}$ S. Stoynev, ${ }^{144}$ J. Strait, ${ }^{144}$ N. Strobbe, ${ }^{144}$ L. Taylor,${ }^{144}$ S. Tkaczyk, ${ }^{144}$ N. V. Tran, ${ }^{144}$ L. Uplegger, ${ }^{144}$ E. W. Vaandering, ${ }^{144}$ C. Vernieri, ${ }^{144}$ M. Verzocchi, ${ }^{144}$ R. Vidal, ${ }^{144}$ M. Wang, ${ }^{144}$ H. A. Weber, ${ }^{144}$ A. Whitbeck, ${ }^{144}$ W. Wu, ${ }^{144}$ D. Acosta, ${ }^{145}$ P. Avery, ${ }^{145}$ P. Bortignon, ${ }^{145}$ D. Bourilkov, ${ }^{145}$ A. Brinkerhoff, ${ }^{145}$ A. Carnes, ${ }^{145}$ M. Carver, ${ }^{145}$ D. Curry, ${ }^{145}$ R. D. Field, ${ }^{145}$ I. K. Furic, ${ }^{145}$ S. V. Gleyzer, ${ }^{145}$ B. M. Joshi, ${ }^{145}$ J. Konigsberg, ${ }^{145}$ A. Korytov, ${ }^{145}$

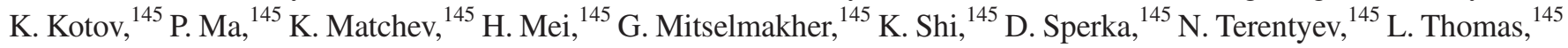
J. Wang, ${ }^{145}$ S. Wang, ${ }^{145}$ J. Yelton, ${ }^{145}$ Y. R. Joshi, ${ }^{146}$ S. Linn, ${ }^{146}$ P. Markowitz, ${ }^{146}$ J. L. Rodriguez, ${ }^{146}$ A. Ackert, ${ }^{147}$ T. Adams, ${ }^{147}$ A. Askew, ${ }^{147}$ S. Hagopian, ${ }^{147}$ V. Hagopian, ${ }^{147}$ K. F. Johnson, ${ }^{147}$ T. Kolberg, ${ }^{147}$ G. Martinez,${ }^{147}$ T. Perry, ${ }^{147}$ H. Prosper, ${ }^{147}$ A. Saha, ${ }^{147}$ A. Santra, ${ }^{147}$ V. Sharma, ${ }^{147}$ R. Yohay, ${ }^{147}$ M. M. Baarmand, ${ }^{148}$ V. Bhopatkar, ${ }^{148}$

S. Colafranceschi, ${ }^{148}$ M. Hohlmann, ${ }^{148}$ D. Noonan, ${ }^{148}$ T. Roy, ${ }^{148}$ F. Yumiceva, ${ }^{148}$ M. R. Adams, ${ }^{149}$ L. Apanasevich, ${ }^{149}$ D. Berry, ${ }^{149}$ R. R. Betts, ${ }^{149}$ R. Cavanaugh, ${ }^{149}$ X. Chen, ${ }^{149}$ S. Dittmer, ${ }^{149}$ O. Evdokimov, ${ }^{149}$ C. E. Gerber, ${ }^{149}$ D. A. Hangal, ${ }^{149}$ D. J. Hofman, ${ }^{149}$ K. Jung, ${ }^{149}$ J. Kamin, ${ }^{149}$ I. D. Sandoval Gonzalez, ${ }^{149}$ M. B. Tonjes, ${ }^{149}$ N. Varelas, ${ }^{149}$ H. Wang, ${ }^{149}$ Z. Wu, ${ }^{149}$ J. Zhang, ${ }^{149}$ B. Bilki, ${ }^{150, q q 9}$ W. Clarida, ${ }^{150}$ K. Dilsiz, ${ }^{150, \text { rrr }}$ S. Durgut, ${ }^{150}$ R. P. Gandrajula, ${ }^{150}$ M. Haytmyradov, ${ }^{150}$ V. Khristenko, ${ }^{150}$ J.-P. Merlo, ${ }^{150}$ H. Mermerkaya,${ }^{150, \text { sss }}$ A. Mestvirishvili, ${ }^{150}$ A. Moeller, ${ }^{150}$ J. Nachtman, ${ }^{150}$ H. Ogul, ${ }^{150, \text { ttt }}$ Y. Onel, ${ }^{150}$ F. Ozok, ${ }^{150 \text {,uu }}$ A. Penzo, ${ }^{150}$ C. Snyder, ${ }^{150}$ E. Tiras, ${ }^{150}$ J. Wetzel, ${ }^{150}$ K. Yi, ${ }^{150}$ B. Blumenfeld, ${ }^{151}$ A. Cocoros, ${ }^{151}$ N. Eminizer, ${ }^{151}$ D. Fehling, ${ }^{151}$ L. Feng, ${ }^{151}$ A. V. Gritsan, ${ }^{151}$ P. Maksimovic,${ }^{151}$ J. Roskes, ${ }^{151}$ U. Sarica, ${ }^{151}$ M. Swartz, ${ }^{151}$ M. Xiao, ${ }^{151}$ C. You, ${ }^{151}$ A. Al-bataineh, ${ }^{152}$ P. Baringer, ${ }^{152}$ A. Bean, ${ }^{152}$ S. Boren, ${ }^{152}$ J. Bowen, ${ }^{152}$ J. Castle, ${ }^{152}$ S. Khalil, ${ }^{152}$ 
A. Kropivnitskaya, ${ }^{152}$ D. Majumder, ${ }^{152}$ W. Mcbrayer, ${ }^{152}$ M. Murray, ${ }^{152}$ C. Rogan, ${ }^{152}$ C. Royon, ${ }^{152}$ S. Sanders, ${ }^{152}$ E. Schmitz, ${ }^{152}$ J. D. Tapia Takaki, ${ }^{152}$ Q. Wang, ${ }^{152}$ A. Ivanov, ${ }^{153}$ K. Kaadze ${ }^{153}$ Y. Maravin, ${ }^{153}$ A. Modak, ${ }^{153}$ A. Mohammadi, ${ }^{153}$ L. K. Saini, ${ }^{153}$ N. Skhirtladze, ${ }^{153}$ F. Rebassoo, ${ }^{154}$ D. Wright, ${ }^{154}$ A. Baden, ${ }^{155}$ O. Baron, ${ }^{155}$ A. Belloni, ${ }^{155}$ S. C. Eno, ${ }^{155}$ Y. Feng, ${ }^{155}$ C. Ferraioli, ${ }^{155}$ N. J. Hadley, ${ }^{155}$ S. Jabeen, ${ }^{155}$ G. Y. Jeng, ${ }^{155}$ R. G. Kellogg, ${ }^{155}$ J. Kunkle, ${ }^{155}$ A. C. Mignerey, ${ }^{155}$ F. Ricci-Tam, ${ }^{155}$ Y. H. Shin, ${ }^{155}$ A. Skuja, ${ }^{155}$ S. C. Tonwar, ${ }^{155}$ D. Abercrombie, ${ }^{156}$ B. Allen, ${ }^{156}$ V. Azzolini, ${ }^{156}$ R. Barbieri, ${ }^{156}$ A. Baty, ${ }^{156}$ G. Bauer,${ }^{156}$ R. Bi, ${ }^{156}$ S. Brandt,${ }^{156}$ W. Busza, ${ }^{156}$ I. A. Cali, ${ }^{156}$ M. D'Alfonso, ${ }^{156}$ Z. Demiragli, ${ }^{156}$ G. Gomez Ceballos, ${ }^{156}$ M. Goncharov, ${ }^{156}$ P. Harris, ${ }^{156}$ D. Hsu, ${ }^{156}$ M. Hu, ${ }^{156}$ Y. Iiyama, ${ }^{156}$ G. M. Innocenti, ${ }^{156}$ M. Klute, ${ }^{156}$ D. Kovalskyi, ${ }^{156}$ Y.-J. Lee, ${ }^{156}$ A. Levin, ${ }^{156}$ P. D. Luckey, ${ }^{156}$ B. Maier, ${ }^{156}$ A. C. Marini, ${ }^{156}$ C. Mcginn, ${ }^{156}$ C. Mironov, ${ }^{156}$ S. Narayanan, ${ }^{156}$ X. Niu, ${ }^{156}$ C. Paus, ${ }^{156}$ C. Roland, ${ }^{156}$ G. Roland ${ }^{156}$ G. S. F. Stephans, ${ }^{156}$ K. Sumorok, ${ }^{156}$ K. Tatar, ${ }^{156}$ D. Velicanu, ${ }^{156}$ J. Wang, ${ }^{156}$ T. W. Wang, ${ }^{156}$ B. Wyslouch, ${ }^{156}$ S. Zhaozhong, ${ }^{156}$

A. C. Benvenuti, ${ }^{157}$ R. M. Chatterjee, ${ }^{157}$ A. Evans, ${ }^{157}$ P. Hansen, ${ }^{157}$ S. Kalafut, ${ }^{157}$ Y. Kubota, ${ }^{157}$ Z. Lesko, ${ }^{157}$ J. Mans, ${ }^{157}$ S. Nourbakhsh, ${ }^{157}$ N. Ruckstuhl, ${ }^{157}$ R. Rusack, ${ }^{157}$ J. Turkewitz, ${ }^{157}$ M. A. Wadud, ${ }^{157}$ J. G. Acosta, ${ }^{158}$ S. Oliveros,${ }^{158}$ E. Avdeeva, ${ }^{159}$ K. Bloom, ${ }^{159}$ D. R. Claes, ${ }^{159}$ C. Fangmeier,${ }^{159}$ F. Golf, ${ }^{159}$ R. Gonzalez Suarez, ${ }^{159}$ R. Kamalieddin, ${ }^{159}$ I. Kravchenko, ${ }^{159}$ J. Monroy, ${ }^{159}$ J. E. Siado, ${ }^{159}$ G. R. Snow ${ }^{159}$ B. Stieger, ${ }^{159}$ J. Dolen, ${ }^{160}$ A. Godshalk, ${ }^{160}$ C. Harrington, ${ }^{160}$ I. Iashvili, ${ }^{160}$ D. Nguyen, ${ }^{160}$ A. Parker, ${ }^{160}$ S. Rappoccio, ${ }^{160}$ B. Roozbahani, ${ }^{160}$ G. Alverson, ${ }^{161}$ E. Barberis,${ }^{161}$ C. Freer, ${ }^{161}$ A. Hortiangtham, ${ }^{161}$ A. Massironi, ${ }^{161}$ D. M. Morse, ${ }^{161}$ T. Orimoto, ${ }^{161}$ R. Teixeira De Lima, ${ }^{161}$ T. Wamorkar, ${ }^{161}$ B. Wang, ${ }^{161}$ A. Wisecarver ${ }^{161}$ D. Wood, ${ }^{161}$ S. Bhattacharya,${ }^{162}$ O. Charaf, ${ }^{162}$ K. A. Hahn,${ }^{162}$ N. Mucia,${ }^{162}$ N. Odell, ${ }^{162}$ M. H. Schmitt, ${ }^{162}$ K. Sung, ${ }^{162}$ M. Trovato, ${ }^{162}$ M. Velasco, ${ }^{162}$ R. Bucci, ${ }^{163}$ N. Dev, ${ }^{163}$ M. Hildreth, ${ }^{163}$ K. Hurtado Anampa ${ }^{163}$ C. Jessop, ${ }^{163}$ D. J. Karmgard, ${ }^{163}$ N. Kellams, ${ }^{163}$ K. Lannon, ${ }^{163}$ W. Li, ${ }^{163}$ N. Loukas, ${ }^{163}$ N. Marinelli, ${ }^{163}$ F. Meng, ${ }^{163}$ C. Mueller, ${ }^{163}$ Y. Musienko, ${ }^{163, k \mathrm{kk}}$ M. Planer, ${ }^{163}$ A. Reinsvold, ${ }^{163}$ R. Ruchti, ${ }^{163}$ P. Siddireddy,${ }^{163}$ G. Smith, ${ }^{163}$ S. Taroni, ${ }^{163}$ M. Wayne, ${ }^{163}$ A. Wightman, ${ }^{163}$ M. Wolf, ${ }^{163}$ A. Woodard, ${ }^{163}$ J. Alimena, ${ }^{164}$ L. Antonelli, ${ }^{164}$ B. Bylsma, ${ }^{164}$ L. S. Durkin, ${ }^{164}$ S. Flowers, ${ }^{164}$ B. Francis, ${ }^{164}$ A. Hart, ${ }^{164}$ C. Hill, ${ }^{164}$ W. Ji ${ }^{164}$ T. Y. Ling, ${ }^{164}$ W. Luo, ${ }^{164}$ B. L. Winer, ${ }^{164}$ H. W. Wulsin, ${ }^{164}$ S. Cooperstein, ${ }^{165}$ O. Driga, ${ }^{165}$ P. Elmer, ${ }^{165}$ J. Hardenbrook, ${ }^{165}$ P. Hebda ${ }^{165}$ S. Higginbotham, ${ }^{165}$ A. Kalogeropoulos, ${ }^{165}$ D. Lange, ${ }^{165}$ J. Luo, ${ }^{165}$ D. Marlow, ${ }^{165}$ K. Mei ${ }^{165}$ I. Ojalvo, ${ }^{165}$ J. Olsen, ${ }^{165}$ C. Palmer, ${ }^{165}$ P. Piroué, ${ }^{165}$ J. Salfeld-Nebgen, ${ }^{165}$ D. Stickland, ${ }^{165}$ C. Tully, ${ }^{165}$ S. Malik, ${ }^{166}$ S. Norberg, ${ }^{166}$ A. Barker, ${ }^{167}$ V. E. Barnes, ${ }^{167}$ S. Das, ${ }^{167}$ L. Gutay, ${ }^{167}$ M. Jones, ${ }^{167}$ A. W. Jung, ${ }^{167}$ A. Khatiwada, ${ }^{167}$ D. H. Miller, ${ }^{167}$ N. Neumeister, ${ }^{167}$ C. C. Peng, ${ }^{167}$ H. Qiu, ${ }^{167}$ J. F. Schulte, ${ }^{167}$ J. Sun, ${ }^{167}$ F. Wang, ${ }^{167}$ R. Xiao, ${ }^{167}$ W. Xie, ${ }^{167}$ T. Cheng, ${ }^{168}$ N. Parashar, ${ }^{168}$ Z. Chen, ${ }^{169}$ K. M. Ecklund, ${ }^{169}$ S. Freed, ${ }^{169}$ F. J. M. Geurts, ${ }^{169}$ M. Guilbaud ${ }^{169}$ M. Kilpatrick, ${ }^{169}$ W. Li,${ }^{169}$ B. Michlin, ${ }^{169}$ B. P. Padley, ${ }^{169}$ J. Roberts, ${ }^{169}$ J. Rorie, ${ }^{169}$ W. Shi, ${ }^{169}$ Z. Tu, ${ }^{169}$ J. Zabel, ${ }^{169}$ A. Zhang, ${ }^{169}$ A. Bodek, ${ }^{170}$ P. de Barbaro, ${ }^{170}$ R. Demina, ${ }^{170}$ Y. t. Duh, ${ }^{170}$ T. Ferbel, ${ }^{170}$ M. Galanti, ${ }^{170}$ A. Garcia-Bellido, ${ }^{170}$ J. Han, ${ }^{170}$ O. Hindrichs, ${ }^{170}$ A. Khukhunaishvili, ${ }^{170}$ K. H. Lo, ${ }^{170}$ P. Tan, ${ }^{170}$ M. Verzetti, ${ }^{170}$ R. Ciesielski, ${ }^{171}$ K. Goulianos, ${ }^{171}$ C. Mesropian, ${ }^{171}$ A. Agapitos, ${ }^{172}$ J. P. Chou, ${ }^{172}$ Y. Gershtein, ${ }^{172}$ T. A. Gómez Espinosa, ${ }^{172}$ E. Halkiadakis, ${ }^{172}$ M. Heindl, ${ }^{172}$ E. Hughes,${ }^{172}$ S. Kaplan, ${ }^{172}$ R. Kunnawalkam Elayavalli, ${ }^{172}$ S. Kyriacou, ${ }^{172}$ A. Lath, ${ }^{172}$ R. Montalvo, ${ }^{172}$ K. Nash ${ }^{172}$ M. Osherson, ${ }^{172}$ H. Saka, ${ }^{172}$ S. Salur, ${ }^{172}$ S. Schnetzer, ${ }^{172}$ D. Sheffield, ${ }^{172}$ S. Somalwar, ${ }^{172}$ R. Stone, ${ }^{172}$ S. Thomas, ${ }^{172}$ P. Thomassen, ${ }^{172}$ M. Walker, ${ }^{172}$ A. G. Delannoy, ${ }^{173}$ J. Heideman, ${ }^{173}$ G. Riley, ${ }^{173}$ K. Rose,${ }^{173}$ S. Spanier, ${ }^{173}$ K. Thapa, ${ }^{173}$ O. Bouhali, ${ }^{174, \text { vvv }}$ A. Castaneda Hernandez, ${ }^{174, \text { vvv }}$ A. Celik, ${ }^{174}$ M. Dalchenko, ${ }^{174}$ M. De Mattia, ${ }^{174}$ A. Delgado, ${ }^{174}$ S. Dildick ${ }^{174}$ R. Eusebi, ${ }^{174}$ J. Gilmore, ${ }^{174}$ T. Huang, ${ }^{174}$ T. Kamon, ${ }^{174, \text { www }}$ R. Mueller, ${ }^{174}$ Y. Pakhotin,${ }^{174}$ R. Patel, ${ }^{174}$ A. Perloff, ${ }^{174}$ L. Perniè, ${ }^{174}$ D. Rathjens, ${ }^{174}$ A. Safonov, ${ }^{174}$ A. Tatarinov, ${ }^{174}$ N. Akchurin, ${ }^{175}$ J. Damgov, ${ }^{175}$ F. De Guio, ${ }^{175}$ P. R. Dudero, ${ }^{175}$ J. Faulkner, ${ }^{175}$ E. Gurpinar, ${ }^{175}$ S. Kunori, ${ }^{175}$ K. Lamichhane, ${ }^{175}$ S. W. Lee,${ }^{175}$ T. Mengke,${ }^{175}$ S. Muthumuni, ${ }^{175}$ T. Peltola, ${ }^{175}$ S. Undleeb, ${ }^{175}$ I. Volobouev, ${ }^{175}$ Z. Wang, ${ }^{175}$ S. Greene, ${ }^{176}$ A. Gurrola, ${ }^{176}$ R. Janjam, ${ }^{176}$ W. Johns, ${ }^{176}$ C. Maguire, ${ }^{176}$ A. Melo, ${ }^{176}$ H. Ni ${ }^{176}$ K. Padeken, ${ }^{176}$ P. Sheldon, ${ }^{176}$ S. Tuo, ${ }^{176}$ J. Velkovska, ${ }^{176}$ Q. Xu, ${ }^{176}$ M. W. Arenton, ${ }^{177}$ P. Barria, ${ }^{177}$ B. Cox,${ }^{177}$ R. Hirosky, ${ }^{177}$ M. Joyce, ${ }^{177}$ A. Ledovskoy, ${ }^{177}$ H. Li, ${ }^{177}$ C. Neu, ${ }^{177}$ T. Sinthuprasith, ${ }^{177}$ Y. Wang, ${ }^{177}$ E. Wolfe, ${ }^{177}$ F. Xia, ${ }^{177}$ R. Harr, ${ }^{178}$ P. E. Karchin,${ }^{178}$ N. Poudyal, ${ }^{178}$ J. Sturdy, ${ }^{178}$ P. Thapa, ${ }^{178}$ S. Zaleski, ${ }^{178}$ M. Brodski, ${ }^{179}$ J. Buchanan, ${ }^{179}$ C. Caillol, ${ }^{179}$ D. Carlsmith,${ }^{179}$ S. Dasu, ${ }^{179}$ L. Dodd, ${ }^{179}$ S. Duric, ${ }^{179}$ B. Gomber ${ }^{179}$ M. Grothe, ${ }^{179}$ M. Herndon, ${ }^{179}$ A. Hervé, ${ }^{179}$ U. Hussain, ${ }^{179}$ P. Klabbers, ${ }^{179}$ A. Lanaro, ${ }^{179}$ A. Levine, ${ }^{179}$ K. Long, ${ }^{179}$ R. Loveless, ${ }^{179}$ V. Rekovic, ${ }^{179}$ T. Ruggles, ${ }^{179}$ A. Savin, ${ }^{179}$ N. Smith, ${ }^{179}$ W. H. Smith, ${ }^{179}$ and N. Woods ${ }^{179}$

(CMS Collaboration) 


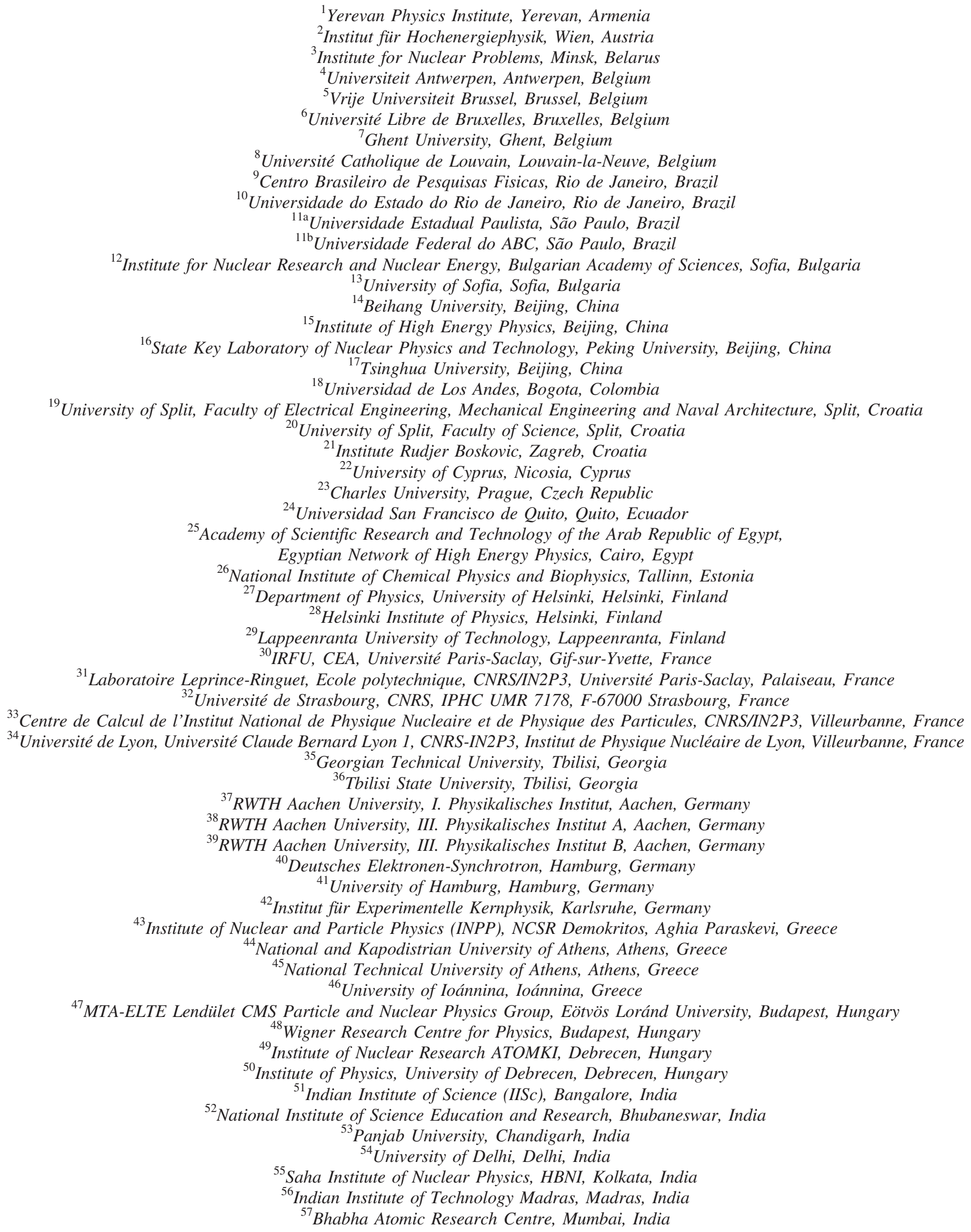




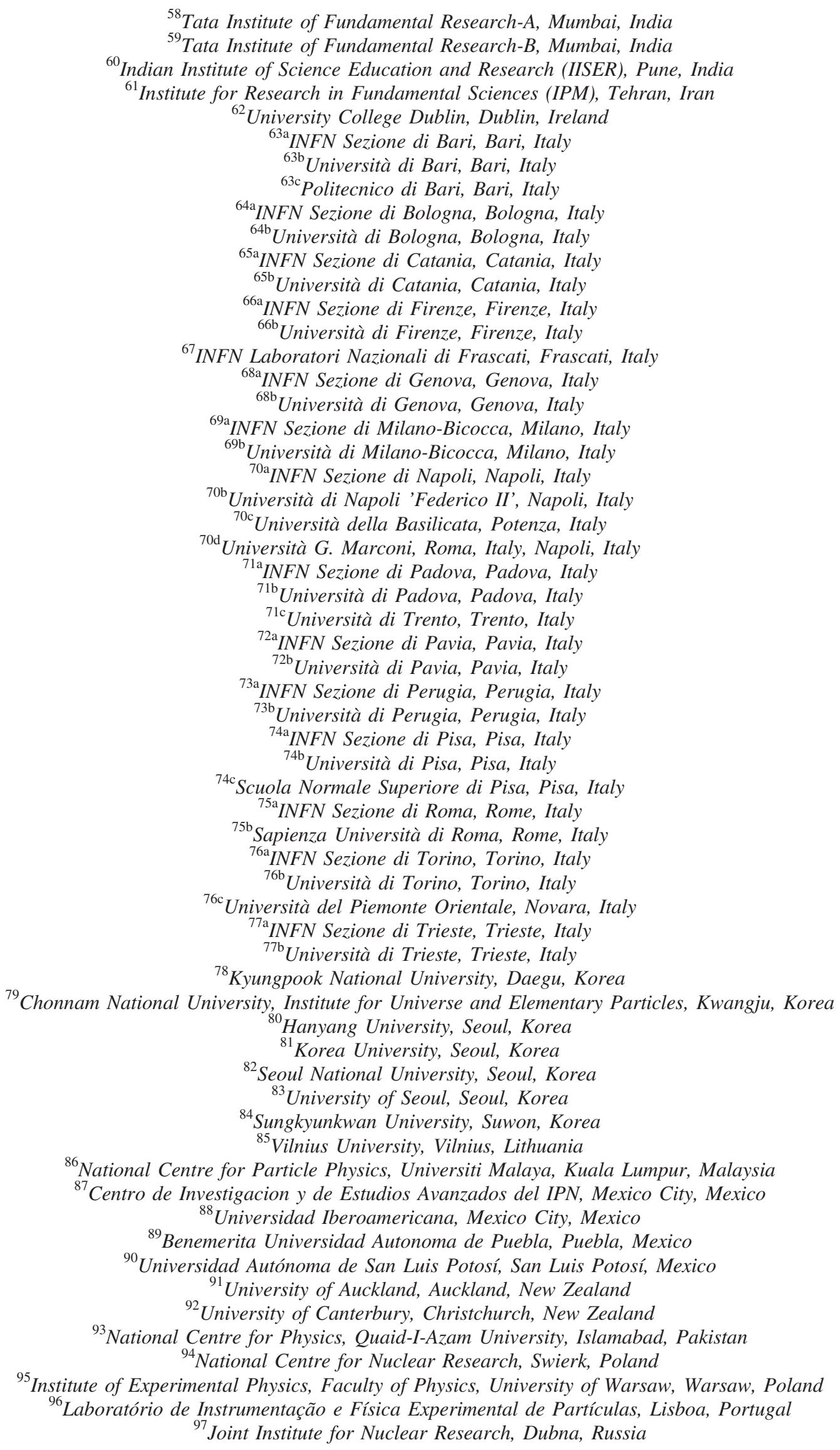




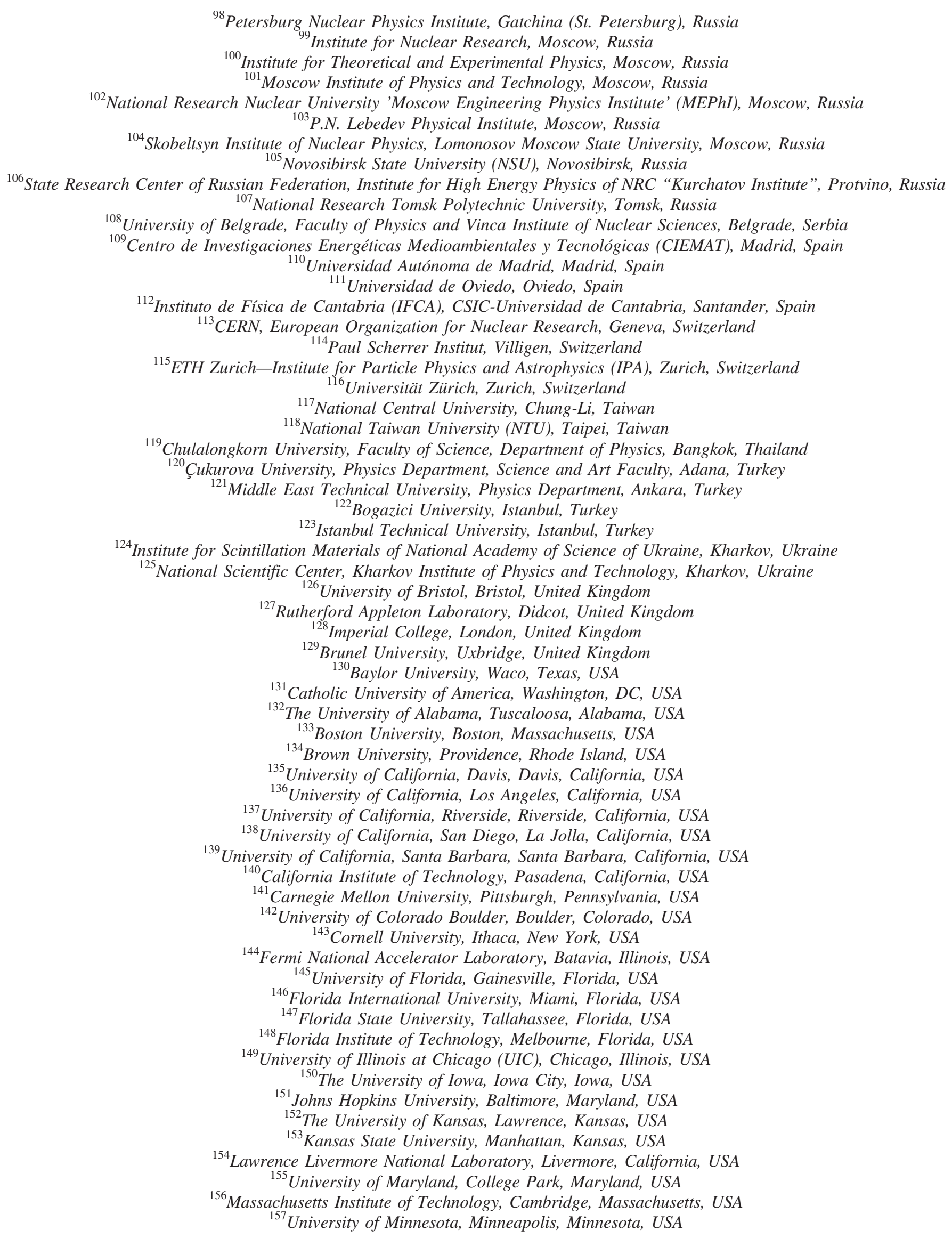




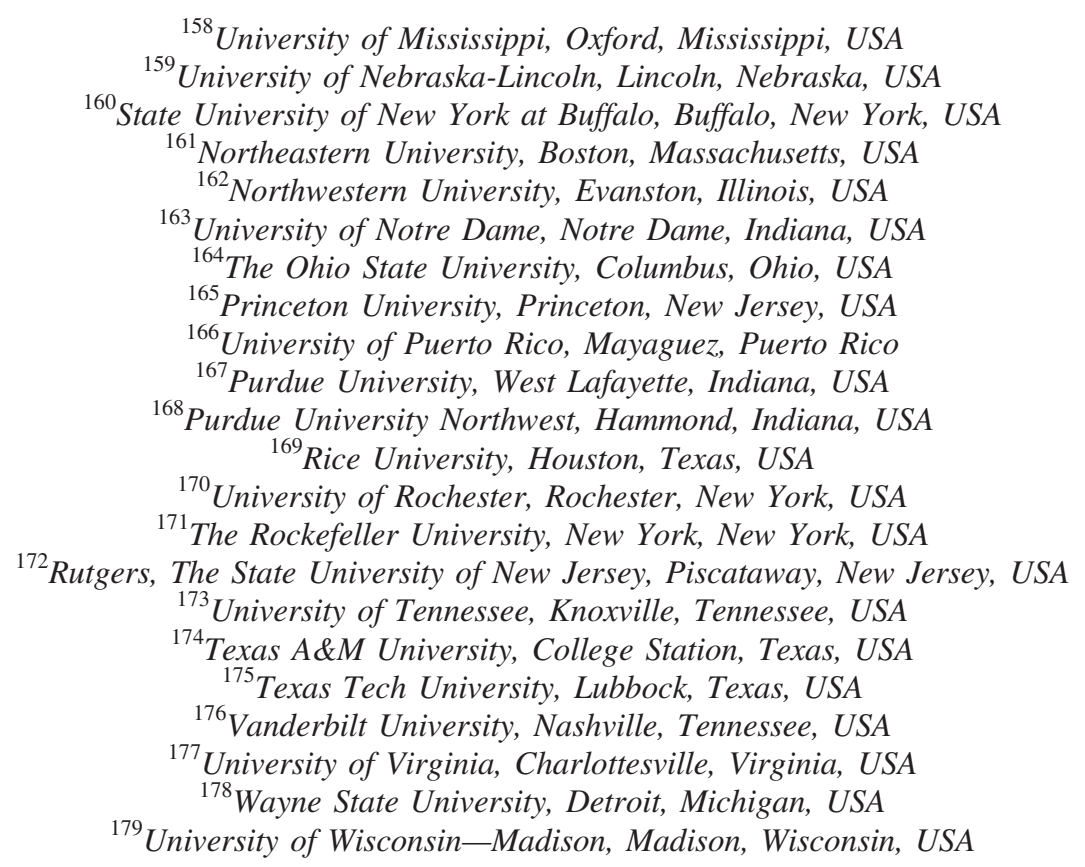

${ }^{\mathrm{a}}$ Deceased.

${ }^{\mathrm{b}}$ Also at Vienna University of Technology, Vienna, Austria.

${ }^{c}$ Also at IRFU, CEA, Université Paris-Saclay, Gif-sur-Yvette, France.

${ }^{\mathrm{d}}$ Also at Universidade Estadual de Campinas, Campinas, Brazil.

${ }^{\mathrm{e}}$ Also at Federal University of Rio Grande do Sul, Porto Alegre, Brazil.

${ }^{\mathrm{f}}$ Also at Universidade Federal de Pelotas, Pelotas, Brazil.

${ }^{\mathrm{g}}$ Also at Université Libre de Bruxelles, Bruxelles, Belgium.

${ }^{\mathrm{h}}$ Also at Institute for Theoretical and Experimental Physics, Moscow, Russia.

${ }^{\mathrm{i}}$ Also at Joint Institute for Nuclear Research, Dubna, Russia.

${ }^{\mathrm{j}}$ Also at Helwan University, Cairo, Egypt.

${ }^{\mathrm{k}}$ Also at Zewail City of Science and Technology, Zewail, Egypt.

${ }^{1}$ Also at British University in Egypt, Cairo, Egypt.

${ }^{\mathrm{m}}$ Also at Department of Physics, King Abdulaziz University, Jeddah, Saudi Arabia.

${ }^{\mathrm{n}}$ Also at Université de Haute Alsace, Mulhouse, France.

${ }^{\circ}$ Also at Skobeltsyn Institute of Nuclear Physics, Lomonosov Moscow State University, Moscow, Russia.

${ }^{\mathrm{p}}$ Also at CERN, European Organization for Nuclear Research, Geneva, Switzerland.

${ }^{\mathrm{q}}$ Also at RWTH Aachen University, III. Physikalisches Institut A, Aachen, Germany.

${ }^{\mathrm{r}}$ Also at University of Hamburg, Hamburg, Germany.

${ }^{\mathrm{s}}$ Also at Brandenburg University of Technology, Cottbus, Germany.

${ }^{\mathrm{t}}$ Also at MTA-ELTE Lendület CMS Particle and Nuclear Physics Group, Eötvös Loránd University, Budapest, Hungary.

${ }^{u}$ Also at Institute of Nuclear Research ATOMKI, Debrecen, Hungary.

${ }^{v}$ Also at Institute of Physics, University of Debrecen, Debrecen, Hungary.

${ }^{\mathrm{w}}$ Also at IIT Bhubaneswar, Bhubaneswar, India.

${ }^{\mathrm{x}}$ Also at Institute of Physics, Bhubaneswar, India.

${ }^{y}$ Also at Shoolini University, Solan, India.

${ }^{\mathrm{z}}$ Also at University of Visva-Bharati, Santiniketan, India.

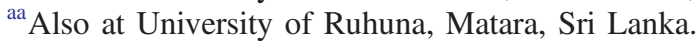

${ }^{\mathrm{bb}}$ Also at Isfahan University of Technology, Isfahan, Iran.

${ }^{\mathrm{cc}}$ Also at Yazd University, Yazd, Iran.

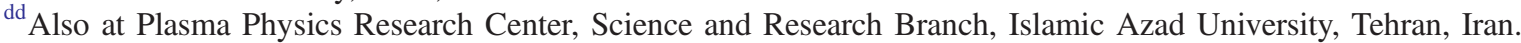

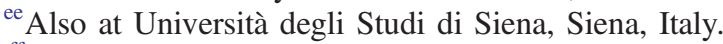

${ }^{\mathrm{ff}}$ Also at INFN Sezione di Milano-Bicocca, Università di Milano-Bicocca, Milano, Italy.

${ }^{\mathrm{gg}}$ Also at International Islamic University of Malaysia, Kuala Lumpur, Malaysia.

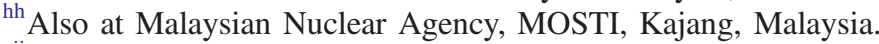

${ }^{i i}$ Also at Consejo Nacional de Ciencia y Tecnología, Mexico city, Mexico.

${ }^{\mathrm{jj}}$ Also at Warsaw University of Technology, Institute of Electronic Systems, Warsaw, Poland.

${ }^{\mathrm{k}}$ Also at Institute for Nuclear Research, Moscow, Russia. 


\footnotetext{
${ }^{11}$ Also at National Research Nuclear University 'Moscow Engineering Physics Institute' (MEPhI), Moscow, Russia.

${ }^{\mathrm{mm}}$ Also at St. Petersburg State Polytechnical University, St. Petersburg, Russia.

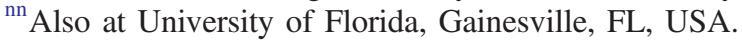

${ }^{\circ o}$ Also at P.N. Lebedev Physical Institute, Moscow, Russia.

${ }^{\mathrm{pp}}$ Also at California Institute of Technology, Pasadena, CA, USA.

${ }^{\mathrm{qq}}$ Also at Budker Institute of Nuclear Physics, Novosibirsk, Russia.

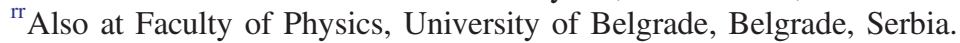

${ }^{\text {ss }}$ Also at INFN Sezione di Pavia, Università di Pavia, Pavia, Italy.

${ }^{t t}$ Also at University of Belgrade, Faculty of Physics and Vinca Institute of Nuclear Sciences, Belgrade, Serbia.

${ }^{u u}$ Also at Scuola Normale e Sezione dell'INFN, Pisa, Italy.

${ }^{v v}$ Also at National and Kapodistrian University of Athens, Athens, Greece.

${ }^{\text {ww }}$ Also at Riga Technical University, Riga, Latvia.

${ }^{\mathrm{xx}}$ Also at Universität Zürich, Zurich, Switzerland.

${ }^{y y}$ Also at Stefan Meyer Institute for Subatomic Physics.

${ }^{\mathrm{zz}}$ Also at Adiyaman University, Adiyaman, Turkey.

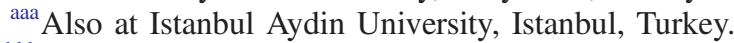

${ }^{b b b}$ Also at Mersin University, Mersin, Turkey.

${ }^{c c c}$ Also at Piri Reis University, Istanbul, Turkey.

${ }^{\text {ddd }}$ Also at Izmir Institute of Technology, Izmir, Turkey.

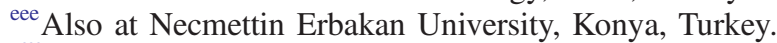

${ }^{\mathrm{fff}}$ Also at Marmara University, Istanbul, Turkey.

${ }^{g g g}$ Also at Kafkas University, Kars, Turkey.

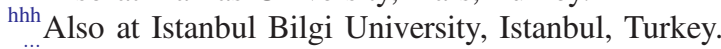

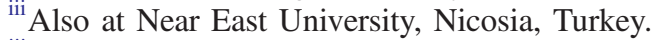

${ }^{j j j}$ Also at Rutherford Appleton Laboratory, Didcot, United Kingdom.

${ }^{k k k}$ Also at School of Physics and Astronomy, University of Southampton, Southampton, United Kingdom.

${ }^{111}$ Also at Monash University, Faculty of Science, Clayton, Australia.

${ }^{\mathrm{mmm}}$ Also at Instituto de Astrofísica de Canarias, La Laguna, Spain.

${ }^{n n n}$ Also at Bethel University, St. Paul, MN, USA.

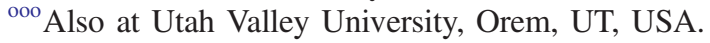

${ }^{\mathrm{ppp}}$ Also at Purdue University, West Lafayette, IN, USA.

${ }^{\mathrm{qqq}}$ Also at Beykent University, Istanbul, Turkey.

${ }^{\mathrm{rrr}}$ Also at Bingol University, Bingol, Turkey.

${ }^{\text {sss }}$ Also at Erzincan University, Erzincan, Turkey.

"tt Also at Sinop University, Sinop, Turkey.

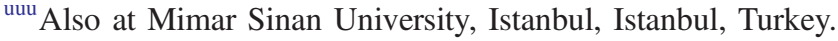

${ }^{\mathrm{vvv}}$ Also at Texas A\&M University at Qatar, Doha, Qatar.

${ }^{w w w}$ Also at Kyungpook National University, Daegu, Korea.
} 